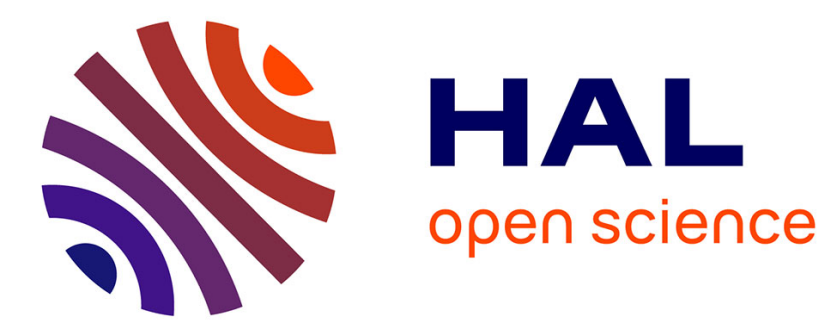

\title{
Les fouilles de sauvetage de Zeugma: un bilan des résultats
}

Catherine Abadie-Reynal

\section{To cite this version:}

Catherine Abadie-Reynal. Les fouilles de sauvetage de Zeugma: un bilan des résultats. Journal of Roman Archaeology (JRA), 2015. hal-01729098

\section{HAL Id: hal-01729098 \\ https://hal.science/hal-01729098}

Submitted on 12 Mar 2018

HAL is a multi-disciplinary open access archive for the deposit and dissemination of scientific research documents, whether they are published or not. The documents may come from teaching and research institutions in France or abroad, or from public or private research centers.
L'archive ouverte pluridisciplinaire HAL, est destinée au dépôt et à la diffusion de documents scientifiques de niveau recherche, publiés ou non, émanant des établissements d'enseignement et de recherche français ou étrangers, des laboratoires publics ou privés. 


\section{JOURNAL OF ROMAN \\ ARCHAEOLOGY}

\section{VOLUME 282015}

$* *$

REVIEW ARTICLES AND LONG REVIEWS,

A SYNTHESIS ON ZEUGMA,

OBITUARIES, AND BOOKS RECEIVED 


\section{EDITORIAL COMMITTEE}

Alan K. Bowman, Brasenose College, Oxford

Katherine M. D. Dunbabin, Lynden, Ontario

Pierre Gros, Aix-en-Provence

John W. Hayes, Oxford

Eugenio La Rocca, University of Rome 1

Carlo Pavolini, Università della Tuscia a Viterbo

Jean-Pierre Sodini, Colombes

Eva Margareta Steinby, Helsinki

Andrew Wallace-Hadrill, Cambridge

\section{ADVISORY COMMITTEE}

T. V. Buttrey, Fitzwilliam Museum, Cambridge

Amanda Claridge, London

Moshe Fischer, Department of Classics, Tel-Aviv University

David L. Kennedy, University of Western Australia

Roger Ling, University of Manchester

Michael Mackensen, Ludwig-Maximilians-Universitaet München

John Matthews, Yale University

Richard Neudecker, Deutsches Archäologisches Institut, Rom

Nicholas Purcell, Brasenose College, Oxford

Isabel Rodà, Universitat Autonòma de Barcelona

Russell T. Scott, Department of Latin, Bryn Mawr College

Cinzia Vismara, Roma

Editor: John H. Humphrey, Portsmouth, Rhode Island

InDesign layout: Claudine Corey, Timothy Madden \& Keith Henry

ISSN 1047-7594 (for the annual journal)

The abbreviation for this journal for purposes of citation is JRA.

This journal is printed on acid-free paper and the signatures are sewn.

Copyright (C) 2015 Journal of Roman Archaeology L.L.C. unless otherwise indicated

Permission to copy may be obtained only direct from JRA ${ }^{\circledR}$, by mail, letter, fax or phone.

E-mail address: jra@JournalofRomanArch.com Phone (USA+) 4016831955

Mailing address: 95 Peleg Road, Portsmouth, Rhode Island 02871, USA Fax (USA+) 4016831975

The Copyright Clearance Center (USA), the Copyright Licensing Agency (UK), and other national Reproduction Rights Organizations are not permitted to authorize copying or to collect fees for so doing.

The opinions expressed in the articles and book reviews published in this journal are those of the authors and not those of the editor or the editorial committee. Reviews are solicited in good faith.

The Journal of Roman Archaeology ${ }^{\circledR}$ has a home page on the World Wide Web, at:

http://www.JournalofRomanArch.com

The home page contains the full table of contents of all published issues of the journal, as well as an index of all books reviewed arranged alphabetically by author, and indices by topic and by site of all articles published. The home page also gives details of titles in the supplementary series (with special offers for individuals). 


\section{Table of contents of fascicule 2 \\ Reviews}

C. L. Sulosky Weaver

A. Nijboer

M. Squire

M. Mogetta

E. Lanza Catti

R. J. A. Wilson

M. Gleba

J.-P. Thuillier

A. Ziolkowski

T. P. Wiseman

J. Packer

E. D'Ambra

D. Woods

M. Beckmann

B. Borg

K. Meinecke

E. Fentress

C. Bruun

E. Dwyer

E. K. Gazda \&

L. J. McAlpine

P. Keegan

C. C. Mattusch

P. F. Bang

A. Van Oyen

A. Corbeill

B. W. Frier

E. Smith

N. Monteix

C. Virlouvet

E. Botte

E. R. Gebhard

J. P. Oleson

W. E. Mierse

M. Feugère

M. MacKinnon

J. P. Oleson

G. Davies

P. Keegan

R. Reece
The indigenous site of Castiglione di Ragusa (Sicily) and its cemeteries in the wake of Greek colonization

The Etruscan world and Rome

Ars reuixit? In search of the ancient artist

A new approach to early urbanization in central Italy

Apulian and Lucanian red-figure pottery and issues of cultural identity

545

The coinage of Iaitas in the context of West Sicily

554

An experimental study of linen versus metal armour

558

Sport grec, spectacle romain?

563

Reading Coarelli's Palatium, or the Sacra via yet again

569

Reading the City

582

Reconstructing Pompey's Theater

587

The short life of Rome's columbaria

591

A new edition of The death of Caligula

594

New drawings of the Column of Marcus Aurelius

596

Roman sarcophagi in context

599

Looking at identity through portraits on sarcophagi

605

Oriental gods on the Caelian Hill

610

Ostian epigraphy in situ

615

The provenance of artifacts discovered on the Vesuvian sites

620

between 1738 and c.1860

The 'Villa of Publius Fannius Synistor' at Boscoreale:

622

putting it all together

The 'graffiti experience' at Pompeii

630

Architecture and luxury on the Bay of Naples

632

An economist approaches Roman economic history 637

Globalisation and material culture: the road ahead 641

Why, how or when the Romans laughed 647

Roman law and the marriage of underage girls 652

Broadening the scope for studying gender 665

Identifier les espaces économiques dans le monde romain 669

Les métiers du port: les saccarii, dockers du monde romain antique $\quad 673$

Exploiting the sea $\quad 684$

An exhibit on Poseidon $\quad 688$

Neoria and navalia: not just about ships $\quad 690$

Textiles in the socio-economic sphere of pre-Roman and Roman Europe 695

Fibules, identités et déplacements individuels ou collectifs 699

dans l'Antiquité

A zooarchaeologist studies animals in the larger picture

(or, on the benefits of keeping your own chickens)

The final word on Roman wooden pumping machinery $\quad 707$

$\begin{array}{ll}\text { The siege as a moral contest } & 709\end{array}$

Women and civic life in Italy and the western provinces 712

$\begin{array}{ll}\text { Money for the Roman army } & 717\end{array}$ 


\section{Table of contents of fascicule 2 (continued)}

M. Pitts

T. J. Derrick

J. Wood

R. Ling

F.-S. Kirch

C. Pavel

C. C. Mattusch

S. de Brestian

J. Edmondson

C. Vismara

J. Stoop

A. Spawforth

M. E. Hoskins Walbank

C. B. Rose

P. Scherrer

R. R. R. Smith

L. Campagna

C. Abadie-Reynal

C. S. Lightfoot

K. Butcher

I. R. Scott

A. U. De Giorgi

J. A. Baird

T. Kaizer

W. E. Mierse

A. J. M. Kropp

A. M. Berlin

A. W. White

S. Sidebotham

J. Patrich

C. P. Elliott

A. Hollmann

D. Woods

R. Lim

K. G. Holum

C. Vanderheyde

J. Balty

C. Foss

J. G. Pedley
Debating the Roman origins of Londinium,

with questions about its material signature

726 from Britain

A lament for the defences of Silchester

"Toichographologie" at AFPMA's 26th colloquium:

painted plaster and stucco from Gaul and elsewhere

Die Nutzung des Raumes in römischen Legionslagern und Kastellen

734

The "palace of the procurator" in Trier:

the promise and predicament of Roman archaeology

Broken but not forgotten: fragments of bronze statues

from the northern limes

Landscape archaeology in Roman Spain

Rural epigraphy in central Spain: a new corpus of Latin inscriptions

In cerca della società africana

751

The 'windmill model' of the Augustan revolution

Cult in Roman Greece (mostly)

Inequality in Roman Corinth

The reliefs from the Julio-Claudian Sebasteion at Aphrodisias

The Kouretes in Ephesos: thoughts on their origin, duties, and engagement in cult and social life

Citizens and kings in the Tomba Bella at Hierapolis

Rappresentatività versus funzionalità: una nuova prospettiva per lo studio delle fontane monumentali dell'Oriente romano

Les fouilles de sauvetage de Zeugma: un bilan des résultats

Zeugma: Packard Humanities Institute excavations

Coins from the French excavations at Zeugma, and a comparison

with the PHI coins

Small finds from the French-Turkish excavations at Zeugma

862

and the study of destruction deposits

The Princeton excavations in Antakya, 1932-40

873

Jebel Khalid: counting sherds, or sherds that count? 877

On the origins of Palmyra and its trade $\quad 881$

Sasanian military architecture on Rome's NE frontier 889

The iconography of Greek divinities in the arts of Central Asia 893

A once and future king 895

The Roman games in Palestine and their reception 902

Surveying the Wadi 'Araba near Aqaba 905

The new archaeological Atlas of Petra, fascicule $1 \quad 908$

A "socio-political and environmental" crisis in Roman Egypt 912

The material side of magic and its archaeological contexts 917

Constantine the Great: a partial account 923

The hippodrome of Constantinople: shaping Byzantine civilization 931

and its cultural imagination

Wealth as a "stethoscope" for studying Late Antiquity 936

Les reliquaires du Proche-Orient et le culte des martyrs $\quad 941$

Les mosaïques des provinces antiques d'Arabie et de Palestine 946

aux époques romaine, byzantine et arabe

Coinage and circulation in Byzantine Palestine 954

The ups and downs of William Stillman, an American 19th-c. 956

archaeological photographer (and much more) 
Table of contents of fascicule 2 (continued)

Response by C. Aranegui Gascó to E. Papi's review of Lixus 3 (JRA 26 [2013] 800-7) 962

Obituary of David Peacock, by S. Keay 965

Obituary of Sheppard Sunderland Frere, by R. J. A. Wilson 968

Obituary of Paul B. Harvey, Jr., by C. E. Schultz 974

BOOKS RECEIVED $\quad 976$

BOOKS REVIEWED IN THIS ISSUE 


\title{
Les fouilles de sauvetage de Zeugma: un bilan des résultats
}

\author{
Catherine Abadie-Reynal
}

Fruit du hasard des publications, coup sur coup viennent de paraître plusieurs ouvrages consacrés aux fouilles de sauvetage de Zeugma. En plus des 5 volumes des fouilles francoturques parus à ce jour, ${ }^{1}$ ce sont les résultats des fouilles menées en 2000 (avec quelques prolongements en 2001), avant la mise en eau du barrage, par une équipe anglaise (Oxford Archaeology) avec financement du Packard Humanities Institute (PHI), qui nous sont livrés: il s'agit d'une publication considérable, produit de la collaboration d'une pléiade de scientifiques. ${ }^{2}$ Même si d'autres livres sont encore à paraître et alors que les recherches se poursuivent dans la partie supérieure du site, ${ }^{3}$ il semble d'ores et déjà possible de tracer un bilan de ces fouilles qui ont révélé au monde la richesse d'un nouveau site, jusqu'alors connu par les textes, ${ }^{4}$ reconnu depuis longtemps, ${ }^{5}$ mais peu exploré par les archéologues. ${ }^{6}$

Il n'est pas question d'envisager, de façon exhaustive, l'ensemble des questions abordées dans ces ouvrages. Nous avons préféré choisir quelques thèmes suffisamment larges pour donner à voir les angles d'approche, les points de vue et les problématiques des équipes qui sont intervenues dans des conditions, il faut le rappeler, très différentes: l'équipe franco-turque a pu fouiller pendant 5 campagnes (de 1996 à 2000), avec un budget limité, mais elle a travaillé sur les deux rives de l'Euphrate, à Séleucie-Zeugma et à Apamée. De son côté, l'équipe du PHI a conduit avant tout une grosse campagne de fouille entre juin et octobre 2000, uniquement à Séleucie-Zeugma, juste avant l'immersion partielle du site.

Les équipes ont cantonné leurs interventions à la partie inférieure de la ville antique (sous la ligne des $389 \mathrm{~m}$ ) qui était directement menacée par la montée des eaux. Il faut garder en mémoire cette contrainte très forte qui oblige à la prudence dans la mesure où les fouilles n'ont pu porter que sur une zone précise du site, tandis que la partie haute de la ville nous échappe à peu près complètement; d'ailleurs, grâce aux rares recherches qui ont pu y être conduites, ${ }^{7}$ elle semble présenter une occupation de nature différente, avec en particulier des zones publiques et des monuments importants.

La nécessité d'établir un bilan, d'autre part, s'impose à nous: les différents ouvrages parus abordent peu ou prou, par des biais différents, les mêmes problématiques et, en ce sens, se font écho, mais les éléments de réflexion et les arguments sont souvent dispersés dans les publications. De plus, pour pouvoir les apprécier, les discuter et parfois les nuancer, il faut toujours rappeler les données matérielles qui fondent les interprétations; or, elles sont, en fonction des chantiers, de qualité variable et quantitativement différentes. Enfin, pour complexifier encore la

1 Ouvrages frequémment cités:

Zeugma I = C. Abadie-Reynal et R. Ergeç, Zeugma I: Fouilles de l'habitat (1). La mosaïque de Pasiphaé (Istanbul 2012);

Zeugma II = A. Barbet (dir.), Zeugma II: Peintures murales romaines (Istanbul 2005);

Zeugma III = C. Abadie-Reynal, Zeugma III: Fouilles de l'habitat (2). La maison des Synaristôsai. Nouvelles inscriptions (TMO 62; Lyon 2012);

Zeugma IV = D. Frascone, Zeugma IV: Les monnaies (TMO 63; Lyon 2013);

Zeugma V = N. Dieudonné-Glad, M. Feugère et M. Önal, Zeugma V: Les objets (TMO 64; Lyon 2013);

Ouvrage fréquemment cité

PHI = W. Aylward (éd.), Excavations at Zeugma, conducted by Oxford Archaeology (Los Altos, CA 2013). antique à l'Asie Mineure. Hommages à Raymond Descat (Bordeaux 2012) 275-300.

D. Kennedy, "Ancient sources for Zeugma (Seleucia-Apamea)," dans id. (éd.), The twin towns of Zeugma on the Euphrates: rescue work and historical studies (JRA Suppl. 27, 1998) 139-62. 


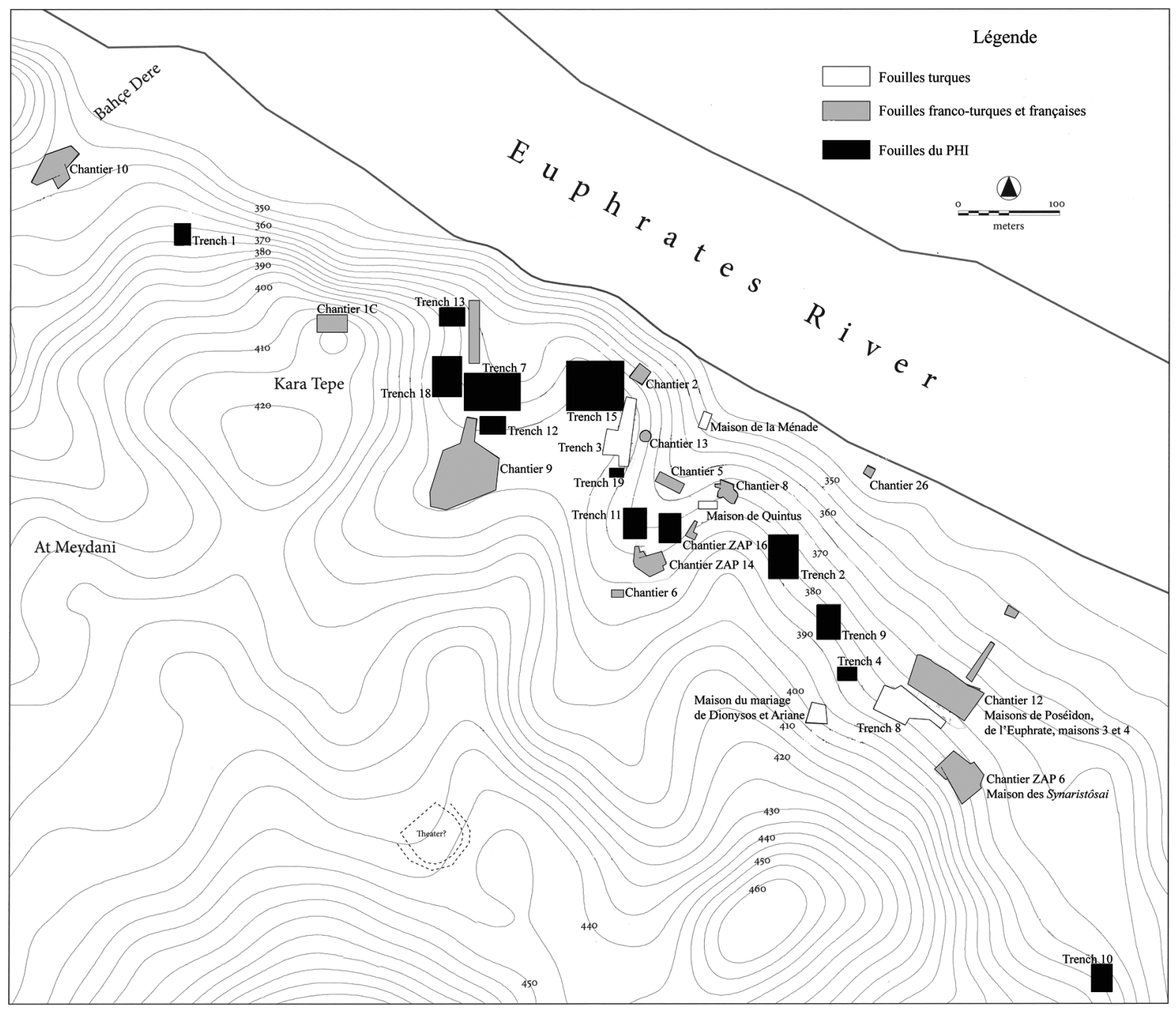

Fig. 1. Situation des différents chantiers (1992-2000) (d'après PHI vol. I, foldout pl. 2, avec modifications).

lecture des résultats, parfois la multiplicité des auteurs mis à contribution entraîne des appréciations différentes à l'intérieur d'une même publication. Ce dialogue et cette confrontation des points de vue sont évidemment féconds, mais ils rendent les résultats globaux de ces travaux parfois difficiles à appréhender.

Les publications des deux équipes, d'autre part, montrent des priorités différentes. L'équipe du PHI, peut-être sous l'influence de la découverte alors toute récente des plus belles maisons et mosaïques de Zeugma dans les fouilles franco-turques, met l'accent sur l'époque romaine, ${ }^{8}$ sans exclure les autres périodes d'occupation du site: elle contribue par exemple excellemment à l'établissement de la typologie des amphores régionales tardives (voir plus bas, p. 842-843); en revanche, l'équipe franco-turque s'attache à étudier le site systématiquement sur la longue durée et souligne les continuités et les fractures qui marquent l'histoire de Zeugma pendant plus de 1000 ans d'occupation du site. L'état d'avancement de la réflexion et sa mise en forme diffèrent aussi d'une équipe à l'autre. La publication du PHI est considérée comme achevée et complète. Aussi, un chapitre introductif présente-t-il l'ensemble des résultats acquis. ${ }^{9}$ Il se très rapidement évoquées et ne représentent que 3 pages sur la vingtaine de pages du texte: voir en particulier Aylward dans PHI vol. 1, 12-13, qui sont consacrées à cette période. Aylward ibid. 
présente comme une synthèse, mais à la lecture de l'ensemble de la publication on se rend compte que des nuances parfois très importantes doivent être apportées à ce texte (voir plus bas, par ex. p. 843). En revanche, l'équipe franco-turque, animée par un autre rythme de publication, se limite à des conclusions pour le moment basées sur des catégories particulières de matériel ou des chantiers précis, sans tenter de synthèse qui, de toute façon, en l'absence de fouilles dans la partie haute du site, serait biaisée. Cependant, grâce à sa plus longue présence sur le terrain, elle dispose d'une grande quantité de matériel et de contextes suffisamment nombreux et riches pour couvrir presque toute la durée d'occupation du site depuis la fin de l'époque hellénistique jusqu'à la fin de l'époque protobyzantine.

Plusieurs thèmes permettent de croiser les points de vue des différentes équipes, tout en évoquant les apports majeurs de ces fouilles. L'évolution du tissu urbain et de l'habitat de Séleucie-Zeugma nous paraît devoir constituer un dossier important et complexe, qui permet de reprendre l'ensemble des données de fouille et de les situer dans un schéma d'évolution sur le long terme. Un thème complémentaire, récurrent dans les publications, parcourt ces travaux, celui de la présence de l'armée sur le site. Il ne s'agira pas de reprendre l'ensemble de la documentation, en grande partie épigraphique, qui permet d'avancer sur ce point - de nombreux travaux ont déjà été faits dans ce sens; ${ }^{10} \mathrm{il} \mathrm{conviendra} \mathrm{plutôt} \mathrm{de} \mathrm{faire} \mathrm{un} \mathrm{bilan} \mathrm{critique} \mathrm{des} \mathrm{traces}$ archéologiques qui peuvent être attribuées aux militaires, et de saisir l'importance réelle et la matérialité de cette présence dans la vie de la cité. Enfin, nous essayerons de réunir les données nouvelles qui permettent de restituer une part des activités économiques de la ville, et de les intégrer à des réseaux d'échanges dont il faudra évoquer les fluctuations et l'étendue. Surtout, au-delà des résultats et des acquis de ces fouilles, il semble utile de mettre l'accent sur les nombreuses questions qui restent ouvertes.

\section{L'évolution du tissu urbain et de l'habitat}

Le problème des fortifications ${ }^{11}$ de la ville de Séleucie-Zeugma, ainsi que celui du franchissement de $\mathrm{l}^{\prime}$ Euphrate, ${ }^{12}$ n'ont pas progressé depuis les conclusions ou les hypothèses avancées par l'équipe franco-turque. De même, l'accord se fait entre les équipes qui ont participé aux fouilles de sauvetage sur le sanctuaire situé au sommet du Belkis Tepe, qui est considéré comme étant extra-urbain. ${ }^{13}$ En revanche, l'évaluation de l'étendue de la ville et le développement de son habitat font l'objet de divergences. ${ }^{14}$

\section{Séleucie à l'époque hellénistique}

L'étendue de la ville hellénistique constitue une première difficulté dans l'appréhension du site. Étant donné les contraintes nombreuses auxquelles se sont heurtées les équipes, on peut

10 Wagner (supra n.6); M. A. Speidel, "Legio IIII Scythica, its movements and men," dans Kennedy (supra n.4) 163-204; H. Devijver, "Commanders and officers of Legio III Scythica," ibid. 205-32; M. Hartmann et M. A. Speidel, "The Roman army at Zeugma: results of new research," dans R. Early et al., Zeugma: interim reports (JRA Suppl. 51, 2003) 101-26.

11 C. Abadie-Reynal et al., "Mission archéologique de Zeugma. Rapport sur la campagne de prospection 1995," AnatAnt 4 (1996) 311-13; ead. et al., "Mission de Zeugma-Moyenne Vallée de l’Euphrate," AnatAnt 5 (1997) 349-51; ead., "L'urbanisme séleucide: l'exemple des sites d'Apamée de l'Euphrate et de Séleucie-Zeugma," dans M.-Th. Le Dinahet (dir.), L'Orient méditerranéen de la mort d'Alexandre au Ier siècle avant notre ère. Anatolie, Chypre, Égypte, Syrie (Nantes 2003) 366-67; repris par Aylward dans PHI vol. 1, 14-15. Görkay (supra n.3) 275-277 reprend l'hypothèse de J. Wagner sur la muraille hellénistique et n'apporte aucun élément nouveau sur les fortifications romaines par rapport à la bibliographie antérieure.

12 Pour un bilan de tous les éléments du dossier et des différentes hypothèses proposées, voir Aylward dans PHI vol. 1, 17-21.

13 Abadie-Reynal 2003 (supra n.11) 367; Aylward dans PHI vol. 1, 27-28; contra Wagner (supra n.6) carte II; Görkay (supra n.3) 281-82.

14 Nous choisissons de garder l'appellation "trench" pour les chantiers conduits sous l'égide du PHI, afin de les distinguer des chantiers de l'équipe franco-turque. 
comprendre que les niveaux hellénistiques aient été difficiles à atteindre et peu étudiés. L'évaluation de l'étendue de la ville à cette époque varie. Les fouilles franco-turques ont livré des points d'accroche solides: elles ont permis de montrer que la limite orientale de la ville s'était fixée au niveau du chantier de la maison des Synaristôsai au plus tard à la fin du IIème ou au début du Ier s. av. J.-C. puisqu'une nécropole qui comprend des tombes de cette époque y a été repérée. ${ }^{15}$ Des niveaux et des structures d'époque hellénistique ont aussi été trouvés dans les chantiers 9,165 et $6 .{ }^{17}$ Quant au chantier 12, il montre que la terrasse n'a été aménagée, au plus tôt, que dans la deuxième moitié du Ier s. av. J.-C. ${ }^{18}$

À l'intérieur même de l'époque hellénistique, on peut ébaucher, à partir de ces données, quelques éléments d'évolution. Les niveaux les plus anciens qui aient été mis au jour paraissent dater de la fin du IIIème ou de la première moitié du IIème s. (chantier 5); ${ }^{19}$ ce chantier semble proche de la limite orientale de la ville à cette époque puisqu'aucun niveau aussi ancien (intégralement constitué, pour la céramique fine, de vernis noir) n'a été trouvé plus à l'est. Les niveaux datant au plus tôt de la deuxième moitié du IIème s. av. J.-C. se trouvent en revanche en plus grand nombre, que ce soit sur les chantiers 5 et 6 ou le chantier 9 . Un point de repère très commode est fourni par la présence régulière de fragments de vases en Sigillée orientale A de forme hellénistique dans ces niveaux. A partir de ces données, nous avons considéré que l'occupation du site était très limitée pendant le début de la période hellénistique, et qu'elle s'est ensuite progressivement développée vers l'est à partir de la fin du IIIème s. (chantier 5) pour concerner, à partir de la deuxième moitié du Ilème s. av. J.-C. au plus tôt, une région relativement étendue, allant du vallon du Bahçe Dere (fig. 1), qui semble avoir constitué la limite ouest dès l'origine, jusqu'à la terrasse qui a abrité la maison des Synaristôsai - même si, à l'intérieur de ces limites, le tissu urbain fut sans doute lâche, comme le montrerait la terrasse de la maison de Poséidon qui semble avoir été mise en place assez tardivement, ou encore l'absence apparente de niveaux de cette époque sur certains chantiers du PHI. ${ }^{20}$

Les données livrées par cette dernière publication semblent apporter des informations complémentaires, confirmant ce processus d'extension du site. L'éditeur, dans son chapitre introductif, s'attache à accorder au moins une importance relative à la Séleucie hellénistique, sur des bases historiques, plutôt qu'archéologiques. ${ }^{21}$ À la lecture du chapitre consacré à l'architecture domestique, on constate, effectivement, que des niveaux hellénistiques ${ }^{22}$ et commagéniens ${ }^{23}$ ont souvent précédé l'habitat romain. ${ }^{24}$ Autrement dit, il apparaît que la ville hellénistique (et commagénienne) a couvert une superficie assez importante, en tout cas à partir de la seconde moitié du IIème s. av. J.-C. ${ }^{25}$

Abadie-Reynal dans Zeugma III, 17-22.

C. Abadie-Reynal et al., "Zeugma-Moyenne Vallée de l'Euphrate. Rapport préliminaire de la campagne de fouilles de 1997," AnatAnt 6 (1998) 392; ead. et al., "Zeugma-Moyenne Vallée de l'Euphrate. Rapport préliminaire de la campagne de fouilles de 1998," AnatAnt 7 (1999) 323; ead. et al., "Zeugma-Moyenne Vallée de l'Euphrate. Rapport préliminaire de la campagne de fouilles de 1999," AnatAnt $8(2000) 323$.

Ead. et al. ibid. 1997, 358 (chantier 6); ead. et al. ibid. 1998, 317 (chantier 5).

Ead., "Chantier 12. Les fouilles françaises dans la Maison de Poséidon (ou Maison 1), la Maison de l'Euphrate (ou Maison 2) et la Maison 4. Années 1999-2000," dans Zeugma I, 209-10.

Cependant, de la céramique de la fin du IVème et au début du IIIème s. aurait été trouvée non loin de là, dans les fondations d'un bâtiment d'archives: voir n.189 et Görkay (supra n.3) 278.

J. Tobin, "The houses: domestic architecture, dated deposits, and finds in context," dans PHI vol. 1, 73 (trench 10), 100 (trench 7B), 102-3 (trench 7C), 106-7 (trench 13), 108 (trench 1).

Aylward dans PHI vol. 1, 9-10.

Tobin (supra n.20) 77 (maison des Casques), 103-4 (maison aux Briques de terre).

Ibid. 93 (maison de la Fontaine), 104 (maison aux Briques de terre).

Ibid. 71-72; voir aussi la carte dans Aylward ibid. 18 où, cependant, ne figure pas la nécropole de la terrasse abritant la maison des Synaristôsai.

Aylward ibid. 10 et 18, fig. 11, qui n'est pas complète puisque, entre autres, la nécropole n'y figure pas (voir n.24). 
Cependant, il est nécessaire de considérer de façon plus approfondie ces informations. La distinction qui est établie par l'équipe du PHI entre les niveaux séleucides (300-64 av. J.-C.) et commagéniens, au premier abord, paraît un moyen commode de préciser les différentes phases de l'évolution du tissu urbain pendant la fin de cette longue période hellénistique. Pourtant, quand on considère les critères qui justifient l'attribution des structures à une phase, on constate qu'ils ne sont pas satisfaisants. En effet, la technique de construction des murs en grand appareil, ${ }^{26}$ considérée comme caractéristique des époques hellénistique et commagénienne, est une particularité qui peut se prolonger bien au-delà de la période considérée. ${ }^{27} \mathrm{La}$ céramique et les monnaies, de leur côté, ne donnent aucune information fiable: un seul groupe de céramique d'époque hellénistique a été publié, ${ }^{28}$ qui est attribué à la seconde moitié du Ilème s. av. J.-C. sur des bases peu solides, comme le souligne P. Kenrick lui-même avec raison. Il s'agit d'un groupe qui pourrait aussi bien appartenir à la seconde moitié du Ilème s. qu'au Ier s. av. J.-C. Autrement dit, il est difficile de distinguer, en l'état actuel des connaissances, les niveaux de la fin de l'époque séleucide et ceux de l'époque commagénienne. Les monnaies n'apportent pas davantage de certitude. ${ }^{29}$ Dans ces conditions, la prudence dicte de continuer à considérer que la ville s'est particulièrement développée à partir de la fin de l'époque hellénistique, entre la deuxième moitié du IIème s. et le Ier s. av. J.-C., sans que l'on puisse préciser davantage.

Il semble certain que les niveaux du début de l'époque hellénistique sont très rares à Séleucie: ils doivent donc être d'étendue limitée et se concentrer probablement autour du point de passage de l'Euphrate et d'un lieu défensif haut, dominant ce franchissement. ${ }^{30}$ Cela dit, à l'heure actuelle nous ne disposons d'aucun niveau archéologique cohérent qui vienne appuyer cette hypothèse. Le développement que connaît ensuite le site est progressif et aurait commencé au plus tard à la fin du IIIème s. (chantier 5). Cependant, il s'affirme davantage, sur le terrain, ultérieurement, quand son extension atteint la terrasse de la maison des Synaristôsai, selon un processus qui mériterait d'être précisé dans sa chronologie et ses différentes étapes. Un point est sûr: cet essor est matérialisé par des niveaux qui incluent des fragments de Sigillée orientale $\mathrm{A}$, une production qui fait son apparition dans la région au cours de la seconde moitié du IIème s. av. J.-C. ${ }^{31}$ Malheureusement, les formes restent très stables, et il est souvent difficile de distinguer des fragments de cette période d'exemplaires du Ier s. av. J.-C.

Or, l'enjeu est important: il s'agit de comprendre les raisons historiques de cette expansion. Deux hypothèses sont en présence, qui ne s'excluent d'ailleurs pas, mais peuvent se renforcer et expliquer l'importance du phénomène. On peut attribuer cet essor urbain à l'apport de populations nouvelles et mettre en parallèle ce développement avec l'abandon, situé à la fin du Ilème ou au début du Ier s. av. J.-C., du site d'Apamée, de l'autre côté de l'Euphrate, ${ }^{32}$ dont la

26 Tobin dans PHI vol. 1, 109.

27 Voir, p. ex., M. Önal, “Fouilles du Musée de Gaziantep dans la Maison de Poséidon (ou Maison 1) et la Maison de l'Euphrate (ou Maisons 2 et 3). Années 1999-2000," dans Zeugma I, 70, fig. 38 (mur entre A2 et A5, maison de Poséidon); Abadie-Reynal dans Zeugma III, 32, fig. 21 (mur entre P4 et P9, maison des Synaristôsai); Tobin dans PHI vol. 1, 71-72.

28 P. Kenrick, "Pottery other than transport amphorae," dans PHI vol. 2, 8-9 (groupe A, provenant du Trench 19).

29 K. Butcher, "Coins and hoards," dans PHI vol. 3, 5.

30 Abadie-Reynal 2003 (supra n.11) 366-7; ead. dans Zeugma III, 13-14.

31 Kenrick dans $P H I$ vol. 2, 8, pour ne citer que la source la plus récente.

32 Contra Aylward 2013a, 22-23, repris par Van den Hoek et Aylward ibid. 232: en s'appuyant en particulier sur Pline l'Ancien qui cite Apamée, les auteurs considèrent que les fortifications d'Apamée ont continué à être en fonction pendant l'époque commagénienne et que le site aurait été abandonné entre 17 et 66 apr. J.-C. Ce point de vue est infirmé par les données de fouille: C. Abadie-Reynal et J. Gaborit, "Le développement urbain en Syrie du Nord: étude des cas de Séleucie et d'Apamée de l'Euphrate," dans La Syrie hellénistique. Colloque Tours (Topoi Suppl. 4, 2003) 153. Après étude rapide du matériel, il apparaît qu'aucune céramique pouvant appartenir sûrement au Ier s. apr. J.-C. n'a été trouvée dans les différents sondages ouverts sur l'ensemble du 
population aurait pu trouver refuge sur la rive droite du fleuve après un épisode violent matérialisé, sur le terrain, par une couche de destruction. ${ }^{33}$ Sans doute aussi peut-on invoquer avec vraisemblance $^{34}$ la contribution ultérieure de la dynastie de Commagène, comme le suggère la découverte, par l'équipe du PHI, de fragments d'inscriptions évoquant la consécration, par le roi Antiochos Ier de Commagène, d'un sanctuaire à Séleucie-Zeugma. ${ }^{35}$ En conséquence, au Ier s. av. J.-C., le tissu urbain de Séleucie-Zeugma est déjà relativement étendu: sa limite orientale restera stable jusqu'à la fin du Ier s. ou au début du IIème s. apr. J.-C. ${ }^{36}$ Sans doute se densifie-t-il, ${ }^{37}$ mais ses limites, et en particulier sa limite orientale, semblent avoir été fixées, pendant plusieurs siècles, avant l'essor de la ville romaine qui entraîne, au IIème s. apr. J.-C., une dernière phase d'extension vers l'est. Il semble donc trop restrictif d'écrire "the life cycle of Seleucia/Zeugma appears to mirror the fortunes of Roma" ${ }^{38}$ Les soubresauts de l'histoire dans la seconde partie de l'époque hellénistique, et peut-être dès la fin du IIIème s., entrent pour une part dans le développement de la ville. Il serait important, pour l'époque hellénistique, de définir de façon plus stricte le rythme d'extension du tissu urbain si on veut l'intégrer dans l'histoire de la région. Pour ce faire, une étude précise de la céramique de cette période, actuellement très mal connue, paraît nécessaire pour fournir des outils d'analyse opérants.

Pour l'habitat, les données concernant l'époque hellénistique sont très parcellaires: l'importance de la cour centrale, parfois avec un péristyle complet ou partiel, a déjà été notée, ${ }^{39}$ et se trouve confirmée par les fouilles du $\mathrm{PHI}^{40}{ }^{4}$ sans qu'il soit possible de compléter ce point: aucun habitat de cette époque n'a, dans les fouilles du PHI, été mis au jour de façon suffisamment complète pour travailler sur la circulation ou les fonctions des pièces. Un seul élément nouveau fait son apparition, qui pourrait être important, mais qui est d'interprétation douteuse: des latrines auraient été trouvées dans la maison de la Fontaine, qui sont attribuées à la période commagénienne ${ }^{41}$ dans un premier temps, puis à l'époque romaine un peu plus loin. ${ }^{42}$ Cette imprécision est regrettable car d'autres latrines ont été repérées sur le site, mais elles semblent toutes appartenir aux dernières phases de fonctionnement des maisons romaines, au moment où l'eau courante était disponible sur le site. ${ }^{43}$ Aucun élément décoratif pouvant remonter à l'époque hellénistique, d'autre part, n'a pu être mis au jour dans les différentes fouilles (mosaïques, enduits peints, par exemple). Même si la disparition du décor peut en grande partie s'expliquer par les lourds remaniements d'époque romaine, cependant, de façon générale (en dehors de quelques grands sites comme Pergame ou Délos) les maisons d'époque

site par l'équipe franco-turque. De plus, l'étude de la nécropole d'Apamée semble confirmer que l'occupation du site connaît un coup d'arrêt qui ne peut être postérieur au milieu du Ier s. av. J.-C. puisqu'aucun unguentarium ovoïde n'a été mis au jour dans ces contextes. L'ensemble de la ville a été abandonné à la fin du IIème ou au début du Ier s. av. J.-C., ce qui n'exclut pas éventuellement qu'un très petit noyau d'occupation ait subsisté au-delà (comme tête de pont, par exemple).

Abadie-Reynal et al. 1997 (supra n.11) 369; ead. et al. 1998 (supra n.16) 403; ead. et al. 1999 (supra n.16) 340; ead. et Gaborit (supra n.32) 151.

Aylward dans PHI vol. 1, 22.

C. Crowther, "Inscriptions on stone," dans PHI vol. 1, 193-203; C. B. Rose, "A new relief of Antiochus I of Commagene and other stone sc ulpture from Zeugma," dans PHI vol. 1, 220-28.

Abadie-Reynal dans Zeugma III, 22.

Terrasse du chantier 12: p. ex., Zeugma I, 209-10.

Aylward dans PHI vol. 1, 22.

C. Abadie-Reynal, "Roman domestic architecture at Zeugma," dans R. Ergeç (éd.), International Symposium on Zeugma: from past to future, 20-22 May 2004 (Gaziantep 2006) 2; ead. dans Zeugma I, 233-34.

40 Tobin dans PHI vol. 1, 77 (maison des Casques) et 109 (maison de la Fontaine).

$41 \quad$ Ibid. 93.

42 Ibid. 109.

43 Önal dans Zeugma I, 100-2 et 136-37 (maison de Poséidon) et 153-54; Abadie-Reynal dans Zeugma I, 195-96 (peu avant le milieu du IIIème s., maison de l’Euphrate); Tobin dans PHI vol. 1, 73-74 (même datation, latrines publiques). 
hellénistique étaient simples et peu décorées. Cette modestie des vestiges apparaît d'ailleurs aussi clairement à Apamée de l'Euphrate. ${ }^{44}$

\section{Zeugma à l'époque romaine}

Toutes les publications s'accordent pour situer l'akmè du site à l'époque romaine, et plus particulièrement dans la première moitié du IIIème $s$. Zeugma a alors l'aspect d'une ville qui atteint son expansion maximale. C'est l'époque où la limite orientale du site est repoussée de plusieurs centaines de mètres vers l'est, ${ }^{45}$ sans doute sous l'effet d'une pression immobilière importante, comme le montreraient certains empiètements sur les zones publiques. Ainsi, le chantier ZAP 14 a livré une maison qui est construite au pied de l'escalier permettant d'atteindre la terrasse supérieure. ${ }^{46}$ Les fouilles du PHI ont aussi montré qu'à cette même époque quelques rares zones n'étaient pas bâties, ${ }^{47}$ qui sont pourtant situées dans des endroits-clefs du site, occupés depuis longtemps. ${ }^{48}$ Donc la densité de l'occupation à l'époque romaine doit encore être évaluée plus finement qu'on ne l'a fait jusqu'à présent; peut-être varie-t-elle en fonction des quartiers.

Il est fait allusion, dans la publication du PHI, à l'existence d'au moins deux trames urbaines, ${ }^{49}$ l'une d'époque séleucide, l'autre du début de l'époque romaine, qui ne sont jamais vraiment détaillées par la suite si ce n'est pour affirmer que la trame hellénistique correspond à celle qui a été repérée à Apamée de $1^{\prime} E_{\text {euphate }}{ }^{50}$ (ce qui semble une affirmation pour le moins rapide). En fait, le relief accidenté du site de Séleucie-Zeugma rend difficile l'organisation d'un urbanisme régulier. Tout au plus, peut-on dire qu'en général les maisons s'adaptent à la topographie du site et sont situées le long de terrasses successives, organisées du nord vers le sud; des rues nord-sud, parfois transformées en escaliers, permettent de suivre les vallons et de rejoindre les différents niveaux de terrasses; des espaces publics permettent de circuler dans le sens est-ouest, le long des terrasses, du côté de l'Euphrate. ${ }^{51}$ En revanche, dans l'état actuel de nos connaissances, rien ne paraît pouvoir supporter l'idée de véritables trames urbaines qui organiseraient la ville (ou du moins certains quartiers), même si, généralement, les rues paraissent orientées nord-sud, ${ }^{52}$ sans véritable régularité, avec, par exemple, un axe nord-est/ sud-ouest $^{53}$ (que semble déjà suivre la maison considérée comme d'époque commagénienne qui précède la maison de la Fontaine); cette rue continuera d'ailleurs à structurer le quartier pendant l'époque romaine.

Les maisons les plus riches et les plus décorées appartiennent toutes à cette période, et plus particulièrement à la première moitié du IIIème s. apr. J.-C. Les fouilles du PHI ont mis au jour, de façon partielle, 13 maisons. Elles permettent de confirmer les éléments majeurs d'évolution qui ont été relevés ailleurs. ${ }^{54}$ Les murs sont généralement en gros appareil au rez-de-chaussée; ils sont surmontés d'élévations en briques de terre crue, ce qui est un mode de construction

44 Voir, p. ex., Abadie-Reynal et al. 1999 (supra n.16) 343-45; ead. et Gaborit (supra n.32) 151.

45 C. Abadie-Reynal et al., "Zeugma. Rapport préliminaire des campagnes de fouilles de 2000," AnatAnt 9 (2001) 297; ead. dans Zeugma III, 22.

46 Voir aussi sur ce problème Abadie-Reynal et al. 2001 (ibid.) 275-92; ead. dans Zeugma I, 197-201 (pièce P1/C6 de la maison de l'Euphrate); ead. dans Zeugma III, 14.

47 Tobin dans PHI vol. 1, 91 (trench 5) et 98 (trench 12).

48 Voir Abadie-Reynal et al. 1998 (supra n.16) 317 (chantier 5 avec une occupation dense d'époque hellénistique et romaine); ead. et al. 1997 (supra n.11) 358 (chantier 6), proches de trench 5; ead. et al. 1998 (supra n.16) 392; ead. et al. 1999 (supra n.16) 323; ead. et al. 2000 (supra n.16) 323 (chantier 9), proche de trench 12.

49 Tobin dans PHI vol 1, 106; repris par Van den Hoek et Aylward dans PHI vol. 1, 232.

$50 \quad$ Abadie-Reynal et al. 1999 (supra n.16) 334-47.

51 Abadie-Reynal et al. 2001 (supra n.45) 275-84.

52 Ead. dans Zeugma I, 215-25; ead. dans Zeugma III, 96-105; Tobin dans PHI vol. 1, 103.

53 Tobin ibid. 82 (trench 2) et 93-97 (trench 11).

54 Abadie-Reynal (supra n.39) 2-5; ead. dans Zeugma I, 233-36; ead. dans Zeugma III, 114-26; Tobin ibid. 72 et 109. 
aussi bien grec que de tradition régionale. ${ }^{55}$ Cet habitat est d'abord organisé autour d'une cour centrale, souvent entourée d'un péristyle, héritée de l'époque hellénistique, mais dont le fonctionnement change. ${ }^{56}$ Les mosaïques et les enduits peints les plus élaborés correspondent à cette phase et renvoient plutôt à la tradition gréco-romaine, même si parfois quelques schémas architecturaux ou quelques éléments du décor peuvent évoquer une tradition locale ou des influences orientales qui réussissent peut-être à percer ou à subsister. ${ }^{57}$ Le développement de la décoration, surtout dans la partie publique de la maison, l'apparition d'un triclinium de type occidental en $U$ ou en $T^{58}$ la modification des circulations introduisant une hiérarchisation des pièces plus marquée, caractérisent l'évolution de ces maisons, en particulier à partir de la fin du IIème s. ${ }^{59}$

Malheureusement, l'interprétation d'une maison, de la fonction des pièces et des circulations ne peut être menée que si l'ensemble de la maison est connu. De ce point de vue, on peut regretter que, malgré les contraintes nombreuses de la fouille, la stratégie du PHI n'ait pas été de fouiller de façon exhaustive quelques maisons et d'établir leur évolution diachronique: les données sur les 13 maisons fouillées ne renouvellent donc guère ce que les maisons du chantier 12 et la maison des Synaristôsai ont montré. ${ }^{60}$ Pourtant, il semble peu vraisemblable, à la lumière de la décoration de ces maisons, des sols mosaïqués et des enduits peints que beaucoup d'entre elles présentent, ${ }^{61}$ de considérer que leur rez-de-chaussée ait pu, à l'instar des maisons rurales syriennes, avoir avant tout une fonction utilitaire, ${ }^{62}$ même juste avant l'attaque sassanide de 252/253 (voir plus bas, p. 829).

On peut regretter, de plus, que l'étude des réseaux d'eau n'ait pas été poussée plus avant. ${ }^{63}$ S'il a été démontré que l'alimentation en eau courante est apparue sur la terrasse du chantier 12 comme dans la maison des Synaristôsai assez tardivement, à la fin du Ilème ou au début du IIIème s. ${ }^{64}$ on aurait aimé pouvoir compléter ces données, vérifier que l'eau courante parvient à la même époque dans les autres parties du site, et confirmer que toutes les maisons ne jouissent pas de ces facilités - seules les plus riches en seraient dotées. ${ }^{65}$ De toutes les maisons mises au jour, il semblerait que seule la maison de la Fontaine ${ }^{66}$ (et peut-être aussi la maison $\mathrm{du}$ Taureau ${ }^{67}$ ) ait connu une alimentation en eau courante, dont pourtant le détail du fonctionnement n'est pas développé, pas plus que l'histoire.

Au-delà de cette impression de richesse qui a frappé les archéologues travaillant sur les niveaux romains de Zeugma, la publication du PHI est parcourue par une idée nouvelle et intéressante, selon laquelle le déclin de ces maisons aurait commencé un peu avant la destruction

Sur l'utilisation très courante, dans le monde grec, de cette technique, voir M.-C. Hellmann, L'architecture grecque, vol. I: Les principes de la construction (Paris 2002) 106-9; contra Aylward dans PHI vol. 1,16 .

quelques exemples, à propos de la mosaïque de triclinium de la maison de Poséidon, dans J.-P. Darmon, “La mosaïque de la pièce P3 (A1) dans la Maison 1 (Maison de Poséidon)," dans Zeugma I, 269; Abadie-Reynal dans Zeugma III, 120.

58 R. Ergeç et M. Önal, “Fouilles archéologiques effectuées en 1998-1999 par le Musée de Gaziantep: la Maison de la Ménade, la Maison d'Okéanos et la Maison aux quadrillages," dans Zeugma I, 26-27 (maison de la Ménade)(?); M. Önal, "Fouilles de sauvetage sur les rives de l'Euphrate. Année 2002," ibid. 53-57 (maison de Quintus), ibid. 140-43 (maison de l'Euphrate); Abadie-Reynal dans Zeugma I, 201-7 (maison de Poséidon); ead. dans Zeugma III, 59 et 119.

59 Ead. dans Zeugma I, 235-36; ead. dans Zeugma III, 114-21.

60 Aylward dans PHI vol. 1, 15-16, repris de façon succincte dans Tobin ibid. 109.

61 Tobin ibid. 75-76, 80, 82, 88-89, 93-95 et 107.

62 Aylward dans PHI vol. 1, 16.

63 Id., ibid. 16-17.

64 Abadie-Reynal dans Zeugma I, 211-13, 221-25 et 234; ead. dans Zeugma III, 112.

65 En revanche, la Maison 4, p. ex., ne comporte pas d'adduction d'eau : ead. dans Zeugma I, 225-32.

66 Tobin dans PHI vol. 1, 94-95.

67 Ead., ibid. 109. 
sassanide du milieu du IIIème s.: elles auraient subi des remodelages, des pièces auraient été divisées. ${ }^{68}$ De plus, la présence d'objets du quotidien suggèrerait un changement de fonction de ces espaces décorés, qui seraient transformés en lieux utilitaires. ${ }^{69}$ Les fouilles francoturques ont également révélé des modifications qui semblent, au premier examen, aller dans ce sens: ainsi le chantier ZAP 14 a-t-il permis de fouiller une maison comportant une pièce avec une mosaïque élaborée, à côté de laquelle on trouve un espace qui, si l'on considère le matériel mis au jour à l'intérieur, pourrait être dédié au stockage des aliments. ${ }^{70}$ Ces remaniements reçoivent des explications différentes suivant les publications: pour l'équipe du PHI, la totalité des modifications (divisions de pièces, bouchages de porte, fermeture d'une aile dans certains péristyles) est attribuée à la présence de militaires cantonnés dans ces maisons. Ils en auraient renforcé la défense en condamnant des passages, tout en assurant leur confort quotidien en divisant les pièces où ils se seraient installés, et en stockant des vivres en prévision de l'attaque. ${ }^{71}$

Cependant, cette hypothèse se heurte à l'apparente facilité avec laquelle la ville aurait été prise, puisqu'aucune trace de combat n'a pu être y trouvée. ${ }^{72}$ De même, nous verrons plus bas (p. 834-835) que la présence militaire ne paraît pas, finalement, aussi évidente, quand on considère les militaria sans a priori. Il existe bien d'autres arguments qui vont à l'encontre de cette explication trop systématique des remaniements. L'abondance des objets luxueux trouvés dans les couches de destruction, ${ }^{73}$ ou encore la présence de restes de nourriture d'exception, ${ }^{74}$ ne constitue pas des signes de déclin et d'occupation militaire. L'emplacement des objets de valeur (principalement des objets métalliques) mis au jour peut difficilement être invoqué pour parler de changement d'usage des pièces: il peut y avoir eu des phénomènes de stockage d'objets que les habitants souhaitaient cacher ou protéger, avant l'attaque, ou que les pillards voulaient emporter à l'issue des opérations militaires. De même, la découverte d'un pithos sur une mosaïque de la maison des Casques, qui aurait contenu des grenades, fruits particulière-

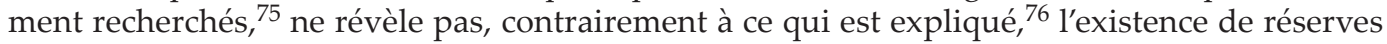
destinées à faire face à l'assaut sassanide! D'autre part, quand il s'agit d'objets de peu de valeur, ou difficilement transportables (céramique, meules, par exemple), il faut prendre en compte l'utilisation fréquente des cours pour les activités domestiques: quand les maisons comportent deux espaces ouverts, une hiérarchie s'établit. Le plus simple des deux abrite la plupart des objets utilitaires, comme nous avons pu le montrer pour la maison de Poséidon. ${ }^{77}$ En revanche, dans les maisons plus modestes, lorsqu'une seule cour est disponible, il est banal, au quotidien, $\mathrm{d}^{\prime} \mathrm{y}$ pratiquer des activités domestiques, comme dans la maison des Casques ${ }^{78}$ ou la maison du Taureau, ${ }^{79}$ sans avoir à invoquer une présence militaire. Enfin, une difficulté supplémentaire, d'ordre archéologique, rend l'interprétation de ces remaniements souvent difficile. Comme on peut le voir dans la maison des Synaristôsai, parfois des réoccupations s'installent directement sur des sols en usage au milieu du IIIème s. (sols mosaïqués ou autres) après un nettoyage soigneux des couches de destruction. ${ }^{80}$ Le matériel (céramique commune le plus souvent)

Aylward ibid. 30; Tobin ibid. 72 et 79 (maison des Casques), 83-84 (maison du Taureau), 88-89 et 95 (maison de la Fontaine), 109-10.

Ead., ibid. 85 (cour de la maison du Taureau et péristyle de la maison des Casques, avec une meule et des vases de stockage et de préparation).

Zeugma V, 304-7.

Aylward dans PHI vol. 1, 24; Tobin ibid. 80, 85 et 109-10.

Aylward, ibid. 31.

Zeugma V, 283; D. Challinor et D. De Moulins, “Charred plant remains," dans PHI vol. 3, 419.

Challinor et De Moulins ibid. 419.

Ibid.

Tobin dans PHI vol. 1, 80.

C. Abadie-Reynal, "La cuisine en Syrie du Nord romaine: premier bilan des données de Zeugma," à paraître.

Tobin dans PHI vol. 1,80 et 85 .

Ibid. 84-85.

Abadie-Reynal dans Zeugma III, 56, 72-74, 79, 90-93, 128-31 (phase 4b); Tobin dans PHI vol. 1, 90 
correspondant à ces réoccupations peut ne pas être bien connu ou n'est pas forcément très différent de celui de la couche de destruction. Quand en plus les murs de la maison romaine n'ont pas été arasés et sont réutilisés, on ne dispose plus véritablement de moyen pour distinguer, à la fouille, une modification antérieure ou postérieure à l'assaut sassanide. Cette difficulté apparaît, par exemple, à propos de l'interprétation de certains niveaux dans la maison de la Fontaine: l'abandon des latrines (et de la fontaine qui a donné son nom à la maison) est considéré comme antérieur à la destruction sassanide parce que ces structures hydrauliques ont été remblayées, ainsi que la mosaïque de la pièce 11E, par de la céramique du milieu du IIIème s., tandis qu'un niveau de sol de mortier a été distingué au-dessus de ce remblai qui comprenait aussi une monnaie d'époque sévérienne. ${ }^{81}$ En fait, à défaut d'autres éléments, cela peut signifier que les latrines ont cessé de fonctionner peu avant ou à l'occasion de l'attaque sassanide. Il ne paraît guère convaincant non plus de conclure de la stratigraphie de la pièce $11 \mathrm{E}$ que le sol de mortier a été mis en place juste avant l'assaut sassanide, d'autant qu'aucune couche de destruction n'est signalée au-dessus de ce sol. ${ }^{82} \mathrm{Il}$ est à craindre que des approximations de ce type ne fassent prendre un certain nombre de réoccupations postérieures à l'attaque sassanide pour des modifications antérieures.

Dans un cas précis, la récurrence d'un remaniement spectaculaire amène à s'interroger sur sa signification. On constate à plusieurs reprises qu'une aile du péristyle de la cour centrale est fermée et transformée en pièce. Une évolution proche a pu être observée en Occident à partir du Ier s., ${ }^{83}$ hors de tout contexte de menace, mais le péristyle est alors fermé par un mur percé de fenêtres, ce qui ne semble pas être le cas à Zeugma autant que l'on puisse en décider à partir de photographies. La fermeture d'une aile du péristyle s'observe dans la maison du Mariage de Dionysos et Ariane, ${ }^{84}$ et dans plusieurs autres maisons fouillées par l'équipe turque du Musée de Gaziantep et le $\mathrm{PHI}^{85}$ qui attribue systématiquement ce remaniement à la nécessité de renforcer dans l'urgence les maisons contre une attaque ennemie. Cependant, plusieurs éléments sembleraient montrer qu'il s'agit plutôt là d'une nouvelle façon d'utiliser le péristyle. Ainsi, dans la maison du Mariage de Dionysos et Ariane, trois niches, évidemment décoratives, sont intégrées dans le mur de fermeture, du côté de l'espace ouvert. Ce mur semble condamner l'accès à la citerne qui se trouvait sous la cour ${ }^{86}$ - autant de caractéristiques bien éloignées d'un souci défensif. D'autre part, la mosaïque de sol qui décore la nouvelle pièce est souvent la plus belle de la maison - ou du moins une des seules mosaïques figurées et la seule qui occupe une aile du péristyle. On peut d'ailleurs, dans certaines maisons de Zeugma, saisir une phase au cours de laquelle une galerie correspondant à une aile d'un péristyle se trouve dotée d'une mosaïque ouvragée, ${ }^{87}$ sans que l'espace ne soit encore fermé. La phase suivante(?) pourrait être marquée par la fermeture de cette aile et sa transformation en pièce à part entière.

(pièce 9H); sur une pratique identique à Antioche et ailleurs, voir É. Morvillez, "L'architecture domestique à Antioche dans l'Antiquité tardive: conservatisme ou modernité?," dans B. Cabouret, P.-L. Gatier et C. Saliou (éds.), Antioche de Syrie. Histoire, images et traces de la ville antique (Topoï Suppl. 5, 2004) 271 et 273.

81 Tobin dans PHI vol. 1, 95.

82 Ibid. 95-96, contrairement à la pièce 11D, par exemple.

83 Sur cette évolution du péristyle, qui tend à constituer un espace autonome avec les pièces de réception, voir $\mathrm{H}$. Dessales, "Du jardin aux jardinières: l'évolution des péristyles domestiques dans l'Italie romaine," dans P. Van Ossel, A.-M. Guimier-Sorbets et M.-C. Marinval (dir.), Archéologie des jardins: analyse des espaces et méthodes d'approche (Nanterre, 8 janvier 2009) (Paris 2014) 26 en particulier.

84 R. Ergeç, "Rescue excavations by the Gaziantep Museum (1992-94)," dans Kennedy (supra n.4) 1-87 et en particulier fig. 5.8.

85 Ergeç et Önal dans Zeugma I, 41-42 (pièce A3, maison d'Okéanos); Tobin dans PHI vol. 1, 79 (pièce $2 \mathrm{H}$, maison des Casques), 83 (pièce $2 \mathrm{M}$, maison du Taureau).

86 Ergeç (supra n.84) 87.

87 Ergeç et Önal dans Zeugma I, 29 (pièce A6 dans la maison de la Ménade); Tobin dans PHI vol. 1, 95 (pièce 11D, maison de la Fontaine). 
On assiste peut-être donc à la constitution progressive d'un nouveau schéma architectural visant à créer, à partir d'une galerie du péristyle, une pièce d'apparat, qui peut parfois précéder le triclinium de la maison. ${ }^{88}$ L'origine de cette création, que l'on peut aussi observer à Antioche, ${ }^{89}$ reste à être précisée et replacée dans un contexte plus large. Ce processus se retrouve, sous une forme différente, dans certains exemples d'habitat tardif à Apamée de $1^{\prime}$ Oronte, ${ }^{90}$ où une longue salle à abside est installée dans une aile du péristyle antérieur par fermeture des entrecolonnements et mise en place d'une mosaïque. La datation de ces modifications pose problème, même si elle semble tardive. À Daphné, une de ces salles allongées paraît pouvoir être datée de la première moitié du Vème s. ${ }^{91}$ Ce schéma architectural, que l'on trouve en Orient, pourrait-il être l'aboutissement d'une nouvelle mode pour les pièces d'apparat, dont on peut voir l'apparition à Zeugma dans la première moitié du IIIème s.?

La fermeture de l'aile d'un péristyle à Zeugma doit être mieux située: si véritablement les cas observés sont antérieurs au milieu du IIIème s., ils précèderaient largement les autres exemples connus de cette pratique en Orient. Mais la fermeture de l'aile du péristyle de la maison du Mariage de Dionysos et Ariane est située à l'époque romaine tardive, ${ }^{92}$ et les autres maisons qui ont subi un tel remaniement portent des traces de passages tardifs. ${ }^{93}$

En tout cas, outre ces problèmes de datation, on ne peut attribuer ces modifications du péristyle, systématiquement, à des préoccupations militaires, sans avoir analysé le phénomène plus avant. L'évaluation des réoccupations postérieures à l'attaque sassanide doit faire l'objet d'un réexamen attentif des données.

\section{Zeugma à l'époque protobyzantine}

À l'occasion de l'étude des niveaux tardifs (principalement IVème-VIIème s.) de la maison des Synaristôsai, nous avons eu l'occasion de faire un bilan des informations fournies par les fouilles franco-turques. ${ }^{94}$ La réoccupation du site commence peut-être dès le IVème s. et progressivement, au cours du Vème et pendant la première moitié du VIème s., il est réinvesti. Il s'agit plutôt d'un habitat rural cette fois-ci, qui se regroupe en quelques noyaux denses dans une occupation discontinue, s'étendant cependant de la limite ouest du site, toujours représentée par le Bahçe Dere, jusqu'à la terrasse de la maison des Synaristôsai à l'est (voir fig. 1). Deux lieux paraissent particulièrement actifs à cette époque et pourraient constituer des sortes de hameaux: le vallon 1 autour du chantier franco-turc 9, plutôt à l'ouest, et la région proche de la maison des Synaristôsai - ce qui n'exclut pas d'autres traces d'occupation, disséminées sur le site. En revanche, les quelques sondages menés dans la partie haute de la ville montreraient qu'elle a été complètement abandonnée dès le Vème s., date à laquelle l'occupation se serait concentrée le long du fleuve. ${ }^{95}$ Finalement ces occupations refluent progressivement au cours du VIème s. et la terrasse de la maison des Synaristôsai est abandonnée vers le milieu du siècle. Certes, après la destruction du milieu du IIIème s. la ville ne recouvre jamais sa prospérité d'antan; ${ }^{96}$ pourtant l'étendue du site reste importante, même si la carte présentée par la publication du $\mathrm{PHI}^{97}$ n'intègre dans la ville des V-VIIème s. ni les chantiers franco-turcs 4, 9 et

88 Ergeç et Önal dans Zeugma I, 26-27 (pièce A1). Sur le lien entre péristyle et triclinium, voir P. Gros, L'architecture romaine, vol. 2. Maisons, palais, villas et tombeaux (Paris 2002) 51. À Antioche, voir la Maison à atrium: A.-S. Martz, Maisons d'Antioche à l'époque romaine: architecture et mobilier archéologique (2001, 71) (https://hal.archives-ouvertes.fr/hal-00749696).

89 Voir la maison du Concours de Boisson (Martz ibid. 28 et 72: première moitié du IIIème s.) ou la maison du bateau des Psychès (ibid. 23 et 72: seconde moitié du IIlème s.); voir aussi peut-être Morvillez (supra n.80) 277, à propos de la maison de la Table servie (fin du IIIème s.).

90 Morvillez (supra n.80) 278-80.

91 Ibid. 278 (maison "aux pavements fleuris"), vers 420-430.

92 Ergeç dans Kennedy (supra n.4) 87.

93 Tobin dans PHI vol. 1, 80 et 84.

94 Abadie-Reynal dans Zeugma III, 134-36.

95 Ead. et al. 2005 (supra n.7) 363.

96 Aylward dans PHI vol. 1, 12, 22 et 110.

97 Ibid. 20, fig. 13. 
10, ni la terrasse de la maison des Synaristôsai, ni l'important chantier ZAP 8 mené par nos collègues turcs, à l'ouest de cette terrasse. ${ }^{98}$ Enfin, l'occupation tardive du Kara Tepe est oubliée. ${ }^{99}$ Cependant, les fouilles du PHI viennent compléter les données des fouilles franco-turques: des niveaux tardifs sont attestés sur presque tous les chantiers ${ }^{100}-$ ces occupations s'échelonnent sur quasiment toute l'étendue du site. De plus, ces fouilles ont livré plusieurs maisons tardives, dont une (trench $7 \mathrm{~B}$ ) présente les caractéristiques d'une maison aisée (cour à péristyle) d'un type encore inconnu à Zeugma, 101 où prédominent les maisons de type rural, organisées autour d'une cour très simple. Malheureusement son analyse architecturale reste très succincte et ne permet pas de mettre en lumière toutes ses caractéristiques et sa place dans une typologie de l'habitat tardif qu'il reste à établir pour la région.

À propos de ces niveaux tardifs, se pose la question du rythme avec lequel le site a été réinvesti après la destruction du milieu du IIIème s. Il a été souligné que les niveaux du IVème s. sont extrêmement rares à Zeugma. ${ }^{102}$ Un habitat de cette époque a été mis au jour dans la cavea du théâtre. ${ }^{103} \mathrm{Il}$ se pourrait aussi que la première phase de réoccupation de la maison des Synaristôsai date de cette époque, ${ }^{104}$ mais il s'agit, en l'absence de matériel, d'une simple supposition. Les fouilles du PHI apportent d'autres éléments: une réoccupation de quelques pièces de la maison des Trésors est attribuée au IVème s.; ${ }^{105} \mathrm{cf}$. la première phase de la maison au Péri style (trench 7B) semble pouvoir être située à la fin du IVème s. ${ }^{106}$ Cependant, les éléments de datation sont ténus: deux fragments de céramique fine pour le premier exemple, une monnaie datée de la deuxième moitié du IVème s. qui ne peut fournir qu'un terminus post quem pour le second. Si ces dates peuvent être retenues, elles montrent que dès les premières réoccupations du site les deux pôles d'occupation à l'ouest et à l'est commencent à se mettre en place. Cependant, comme nous l'avons vu (p. 829-30), il se pourrait aussi que des réoccupations discrètes de maisons romaines puissent appartenir à cette période. Le processus de réinvestissement du site demande encore à être précisé, mais il est, à l'évidence, beaucoup trop rapide d'affirmer qu'avant la fin du Vème s. on ne perçoit pas véritablement de réoccupation. ${ }^{107}$

La ville protobyzantine se développe largement au Vème s., dans la partie inférieure du site comme l'illustrent les fouilles franco-turques ${ }^{108}$ aussi bien que les différents chantiers du PHI. ${ }^{109}$ Pourtant, il faut souligner, pour nuancer l'importance de ce développement, que la partie supérieure du site paraît avoir été en partie ou complètement abandonnée pendant cette période. ${ }^{110} \mathrm{D}^{\prime}$ autre part, à partir du Vlème s. il semble que l'activité se soit ralentie progressivement: certaines zones sont abandonnées dès le milieu du siècle. C'est le cas pour la terrasse de la maison des Synaristôsai, ${ }^{111}$ ainsi peut-être que pour la maison sud-ouest ${ }^{112}$ et le chantier

M. Önal et Y. Yavaş, "Late Roman or Byzantine modifications to the residential area south of the Poseidon, Euphrates and Mars villas," dans Early et al. (supra n.10) 51-55.

Abadie-Reynal et al. 1997 (supra n.11) 349-51; ead. 2003 (supra n.11) 367; ead. dans Zeugma III, 13.

Aylward dans PHI vol. 1, 20, fig. 13 où en particulier le chantier de la maison des Synaristôsai n'est pas figuré, ce qui restreint l'étendue de la ville protobyzantine; Tobin ibid. 72 (trenches 1, 4-5, 7, 9-11 [11 pas mentionné en 72, mais signalé en 96], 12) et 98 (trench 19).

Tobin ibid.100-2.

Abadie-Reynal et al. (supra n.7) 363; C. Abadie-Reynal, A.-S. Martz et A. Cador, "Late Roman and Byzantine pottery in Zeugma: groups of the beginning of the 5th century," dans B. BöhlendorfArslan, A. Osman Uysal et J. Witte-Orr (éds.), Çanak. Late antique and Medieval pottery and tiles in Mediterranean archaeological contexts (Byzas 7, 2007) 182-83.

Abadie-Reynal et al. (supra n.7) 358-59.

Ead. dans Zeugma III, 128-31.

Tobin dans PHI vol. 1, 90.

Ibid. 100.

Aylward dans PHI vol. 1, 22; contra Tobin ibid. 100.

Abadie-Reynal et al. (supra n.7) 364 pour un bilan rapide.

Tobin dans PHI vol. 1, 72, 74, 90-92, 96, 98, 100-3, 108 et 110.

Abadie-Reynal et al. (supra n.7) 363.

Ead. dans Zeugma III, 135-36.

Tobin dans PHI vol. 1, 92. 
appelé trench $4 .{ }^{113}$ Mais on constate aussi, ailleurs, des traces de destruction violente qui seraient datées du milieu du VIIème s. ${ }^{114}$ La rétractation du tissu urbain est donc lente et irrégulière. Ces remarques viennent compléter celles que nous avons pu présenter à propos de la répartition spatiale des céramiques fines tardives. ${ }^{115}$ Nous avons pu déterminer que la plupart des exemplaires de ces productions, datant de la fin du VIème ou du VIIème s., provenaient du chantier 9. On peut maintenant ajouter que les fouilles du PHI confirment la présence, dans cette région du site, du dernier noyau important d'habitations: c'est très majoritairement dans cette région que se situent les chantiers où la couche de destruction du VIIème s. a été repérée (trenches 7 et 12).

\section{L'armée et la guerre à Zeugma}

La question de la présence de l'armée à Zeugma, juste avant l'attaque sassanide, parcourt l'ensemble de la publication des fouilles du PHI et paraît orienter un certain nombre d'interprétations présentées (voir, p. ex., plus haut, p. 829), même si un accord au sein de l'équipe ne semble pas avoir été trouvé sur le sujet. On connaît depuis longtemps la place importante qu'a occupée l'armée sur le site: les textes anciens et l'épigraphie évoquent la présence en particulier de la legio IIII Scythica. Les fouilles du PHI ont ajouté quelques témoignages supplémentaires. ${ }^{116}$ Les travaux de l'équipe suisse auraient pu permettre de préciser le processus d'implantation de la légion à Zeugma puisque les traces d'un camp temporaire, occupé quelques semaines, ont été mises au jour au niveau du mur de barrage. ${ }^{117}$ Par la suite, dans la partie nord-ouest du site, à At Maydanı, les traces d'une base militaire de transit, de dimensions réduites, auraient été trouvées: ${ }^{118}$ de puissants murs sont interprétés comme les vestiges d'une muraille appartenant à cet ensemble; malheureusement aucun détail des fouilles, plan des structures ou catalogue exhaustif du matériel mis au jour n'est fourni pour appuyer ces affirmations. Dans l'attente d'une publication plus complète, le problème du camp de la légion reste donc entier.

La légion a stationné à Zeugma pendant la seconde partie du Ier et le IIème s. La frontière de l'Empire romain fut repoussée vers l'est à la fin de cette période; après 195, la legio IIII Scythica a peut-être, en partie en tout cas, suivi ce déplacement, ${ }^{119}$ tandis que des militaires de cette unité furent postés à Doura Europos où ils sont attestés encore en $254 .{ }^{120}$ Dans ces conditions, une présence militaire importante est-elle (comme le laisse entendre à plusieurs reprises la publication du PHI) envisageable à Zeugma au milieu du IIIème s., au-delà de la présence de quelques détachements? ${ }^{121}$

En l'absence du camp de la légion, cette question peut être abordée par le biais du rapport de la ville et de ses habitants avec l'armée. Pour reprendre les affirmations répétées dès le chapitre introductif de la publication du PHI, l'armée était très présente à Zeugma, dans la ville même, juste avant les destructions du milieu du IIIème s.; des soldats auraient même été cantonnés dans les maisons pour attendre l'attaque sassanide et conduire la résistance ${ }^{122}$ - ce qui

113 Ibid. 86.

114 Ibid. 72, 93, 98-99 et 101-2.

115 C. Abadie-Reynal, “Les sigillées africaines à Zeugma," dans F. Baratte et al. (éds.), Mélanges JeanPierre Sodini (Tr\&Mém 15, Paris 2005) 535.

116 Voir en dernier lieu, avec un rappel de la bibliographie antérieure, C. Crowther dans PHI vol. 1, 203-4; M. Hartmann et M. A. Speidel, "Military installations at Zeugma: an overview of the Swiss archaeological investigations, 2001-2003," dans PHI vol. 3, 385-90.

117 Hartmann et Speidel (supra n.10) 103-5; iid. dans PHI vol. 3, 381.

118 Iid. dans PHI vol. 3, 383-85.

119 Speidel (supra n.10) 174.

120 Ibid. 175; H. Elton, "Zeugma's military history in light of the rescue excavations," dans PHI vol. 3, 377.

121 L'argument selon lequel le capricorne que l'on trouve sur le revers de monnaies frappées à Zeugma sous le règne de Philippe l'Arabe serait le symbole de la legio III Scythica (Speidel 1998 [supra n.10] 175) est actuellement remis en question: voir en dernier lieu Zeugma IV, 217.

Aylward dans PHI vol. 1, 23-24; repris par Tobin ibid. 79, 85 et 110. 
expliquerait les remaniements observés, ${ }^{123}$ ainsi que la présence de militaria qui viennent majoritairement des couches de destruction du milieu du IIIème s. ${ }^{124}$

Cette hypothèse mérite d'être examinée plus longuement. Elle n'est pas sans poser problème. Tout d'abord, l'estimation de l'importance des militaria dans ces niveaux varie selon les auteurs. Ces objets sont considérés comme abondants ${ }^{125}$ dans le chapitre introductif de la publication du PHI mais aussi dans les fouilles franco-turques; ${ }^{126}$ en revanche, d'autres collaborateurs du PHI les jugent peu nombreux. ${ }^{127}$ Malgré ces divergences, un point semble faire l'unanimité: ces objets ont souvent été découverts regroupés dans des contextes précis. Les fouilles franco-turques ont montré que la maison de Poséidon en recelait un grand nombre. ${ }^{128}$ De même, dans les fouilles du PHI une répartition spatiale très particulière a été mise en évidence: la plupart des militaria sont concentrés dans quelques contextes limités (trenches 2, 9 et 18). ${ }^{129}$ Au-delà de ces constatations, il est fondamental d'expliquer la présence de ces objets quand on veut comprendre quelle place occupaient les militaires dans la ville avant l'assaut sassanide.

Pour ce faire, il faut regarder attentivement la nature de ces regroupements. On peut déterminer plusieurs situations: dans la maison de Poséidon, les militaria proviennent de la maison la plus riche qui ait été fouillée; d'autre part, aucune trace significative de déclin de la maison juste avant l'assaut sassanide n'a été observée. Les militaria pourraient alors s'expliquer par une occupation militaire "renforcée", 130 exigée par la présence, dans la maison, d'un éminent personnage et de fortes sommes d'argent. ${ }^{131}$ L'ensemble des objets provient de la partie domestique de la maison où ils auraient été entreposés. Dans les fouilles du PHI, les militaria ont été trouvés en revanche majoritairement dans des maisons plus modestes (maison des Casques, maison du Taureau, maison des Trésors et surtout maison de la Brique de terre crue). On peut remarquer que l'histoire de ces maisons varie: si la maison des Casques et celle du Taureau ont subi des remaniements et des cloisonnements de pièces ${ }^{132}$ avant l'assaut sassanide, en revanche la maison de la Brique de terre crue, qui a livré le plus de militaria de ces fouilles, ${ }^{133}$ ne présente aucune trace de modifications de ce type. ${ }^{134}$ De même, la maison des Trésors, qui a aussi fourni un nombre important de militaria ${ }^{135}$ ne révèle que peu de remaniements associés à cette période.

Donc on ne peut associer automatiquement l'abondance des militaria avec des traces de remodelage des maisons, attribuées à la présence de soldats. Il faut à chaque fois examiner en détail la façon dont se présentent les dépôts d'armes et leur composition. I. Scott suggère que les militaria fragmentaires venant de la maison des Trésors pourraient être les vestiges d'un combat. ${ }^{136}$ Dans la maison des Casques, les objets métalliques sont regroupés en deux lots qui contiennent aussi des objets du quotidien. Scott en déduit qu'ils appartiennent à des soldats cantonnés dans la maison. Pourtant, on peut noter que, dans les moments troublés qui précèdent une destruction, de nombreux pillages se déroulent, qui entraînent aussi le déplacement et le regroupement d'objets: soit leur propriétaire les rassemble dans l'espoir de les emporter

Sur le sujet et certains doutes soulevés par cette interprétation, voir plus haut, p. 829-30.

Abadie-Reynal dans Zeugma III, 364 et 369.

Aylward dans PHI vol. 1, 24.

Zeugma V, 247-49.

I. Scott, "Ironwork," dans PHI vol. 3, 181; id., "Arms, armor, and other military objects," ibid. 353 et 370; Elton, ibid. 78.

Zeugma V, 248 et 282-83.

Scott dans PHI vol. 3, 366-69.

Zeugma V, 248.

M. Önal, Zeugma tetradrachm hoards (Gaziantep 2008) VI-VII.

Voir plus haut p. 829-30 sur de possibles réserves.

Scott dans PHI vol. 3, 368-69.

Tobin dans PHI vol. 1, 105.

Scott dans PHI vol. 3, 367-68.

Ibid. 370. 
avec lui, soit les pillards les réunissent avant de les emporter, mais ils en sont empêchés. ${ }^{137}$ On doit aussi envisager le cas des militaria endommagés, hérités ou peu fonctionnels, ${ }^{138}$ qui constituent sans doute une partie des objets liés à la guerre qui ont été trouvés dans les fouilles. La nature de la présence militaire à Zeugma au milieu du IIIème s. est donc complexe: des nuances doivent être apportées à une analyse trop simpliste du matériel.

Cela nous amène à considérer la façon dont se sont déroulés les événements qui ont entraîné la destruction de Zeugma. La couche cendreuse associée par les différentes équipes à cet épisode dramatique a pu être observée sur de nombreux chantiers. ${ }^{139}$ Elle se caractérise par l'absence complète de restes humains ou même d'animaux qui auraient perdu la vie pendant cet assaut. L'abandon assez général de la ville à l'issue de cette destruction, d'autre part, prouve que les habitants n'ont pu revenir enterrer les morts. Ont-ils été massacrés dans la campagne autour de la ville? Ont-ils été asservis? En tout cas, au moment de l'assaut la ville avait été en grande partie désertée par ses habitants qui connaissaient bien le sort que les Sassanides réservaient aux vaincus.

Les traces de combats sont pour le moins rares en ville; ${ }^{140}$ les militaires, s'il y en avait, ne se sont donc pas retranchés dans les maisons. Aucune résistance n'a été opposée, ce qui explique, en partie, l'étendue des dégâts. En revanche, certains éléments prouvent que les habitants se sont préparés à l'attaque, alors même que la plupart des modifications apportées aux maisons ne semblent pas ressortir d'une volonté de renforcer leur défense (voir plus haut, p. 829-30): ainsi le char de la maison de Poséidon a-t-il probablement été démonté et rangé dans les péristyles et les pièces adjacentes. ${ }^{141}$ De même, quelques trésors ont été cachés (voir plus bas, p. 836-37). On peut imaginer une évacuation rapide, en quelques heures, de la ville, par des habitants qui espéraient revenir. Cette hâte expliquerait que beaucoup d'objets de valeur, encombrants, aient été laissés dans les maisons. D'ailleurs, la maison de Poséidon, qui a livré le plus de militaria, est aussi la maison qui a permis de découvrir le plus d'objets métalliques en général. ${ }^{142}$ La même constatation peut être faite dans les fouilles du PHI: dans la maison des Casques et dans la maison du Taureau de nombreux objets de métal étaient regroupés, ${ }^{143}$ beaucoup d'entre eux ne faisant pas partie de la catégorie des militaria. Ces derniers, finalement, par leur répartition spatiale, ne se démarquent guère des autres objets de valeur; leur regroupement pourrait souvent ressortir du même processus. Cela permet peut-être de concilier deux constatations apparemment contradictoires: la présence assez importante des militaria mis au jour, et l'absence de traces de combats dans la ville.

L'accord est fait pour dater ces couches de destruction des années 252/253 144 ou peut-être 253/254. ${ }^{145}$ En fait, il n'existe aucune continuité stratigraphique entre les différents chantiers, qui viendrait corroborer la stricte contemporanéité de toutes ces couches. Il s'agit d'un a priori commode, accepté par toutes les équipes, qui s'appuie sur le matériel céramique trouvé en

Voir à ce sujet Zeugma V, 250-51 n 1413, 255 n 1432, 282-83. Cette hypothèse est évoquée dans la publication du $P H I$ à propos du regroupement des objets en fer: Scott, PHI vol. 3, 190.

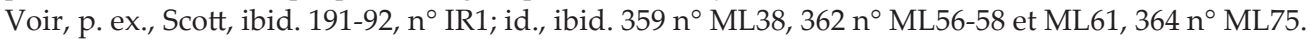
Önal dans Zeugma I, entre autres 122, 134 (maison de Poséidon), 140, 143 et 149 (maison de l'Euphrate); Abadie-Reynal dans Zeugma III, 36-37 (maison des Synaristôsai) et 127-28; Tobin dans PHI vol. 1, 79-80 (maison des Casques), 84 (maison du Taureau), 88 (maison des Tesselles), 89-90 (maison des Trésors), 95-96 (maison de la Fontaine), 106 (maison de la Brique crue), 108 (pièces de la Terrasse supérieure).

140 Seule exception peut-être: Scott dans PHI vol. 3, 370. Sur l'apparente absence de défenseurs dans la ville, Aylward dans PHI vol. 1, 30.

141 Zeugma V, 238-40.

142 Ibid. 282-93.

143 Scott dans PHI vol. 3, 190.

144 M. Sartre, D'Alexandre à Zénobie. Histoire du Levant antique. IVe siècle av. J.-C. - IIIe siècle ap. J.-C. (Paris 2001) 967-71; Abadie-Reynal dans Zeugma I, 237 et Zeugma III, 127-28; Aylward dans PHI vol. 1, 9, 12 et 29-30. 
abondance dans ces niveaux, et sur les monnaies tout aussi nombreuses dans ces couches de destruction. Cela nous amène à nous interroger sur le déroulement de cet assaut sassanide qui semblerait le fait d'un escadron de cavaliers, détaché de l'armée principale. ${ }^{146}$ Certes, nous avons vu que la résistance semble avoir été faible. De plus, il a été remarqué à plusieurs reprises dans les fouilles du PHI que le pillage a été plus ou moins intense suivant les chantiers. ${ }^{147}$ Toutes ces remarques peuvent confirmer que seule une petite unité de cavaliers a pillé la ville. Pourtant, quand on considère l'extension des zones de destruction qui couvrent véritablement tout le site, on peut s'étonner d'un tel pouvoir de destruction et d'une telle volonté de ravager la ville systématiquement, et non simplement de la piller. Un tel épisode est-il vraisemblable?

Cette question, qui n'est jamais évoquée dans les publications, mérite d'être soulevée d'autant que les éléments de datation de ces couches de destruction restent peu significatifs. La contemporanéité de ces niveaux est basée sur les données chronologiques apportées principalement par la céramique et les monnaies. La céramique de ces niveaux commence, certes, à être bien connue (voir plus bas, p. 839). Pourtant, il s'agit avant tout de céramique commune qui n'est pas datée précisément. En revanche, les céramiques fines, qui pourraient apporter des éléments plus précis de datation, sont rares sur le site ${ }^{148}$ à cette époque. Les monnaies paraissent apporter des éléments de datation plus fiables et leur nombre important donne sa pleine signification à ce matériel. Quelles que soient les couches de destruction prises en compte, les monnaies les plus récentes datent, au plus tard, de $253 .{ }^{149}$ Cela dit, à ce moment-là, la plupart des ateliers monétaires régionaux ont cessé de frapper. ${ }^{150}$ L'atelier de Zeugma en particulier s'est arrêté sous Philippe l'Arabe ${ }^{151}$ pour une raison qui nous échappe mais qu'il serait important de déterminer. La menace sassanide se fit-elle plus pressante? ${ }^{152} \mathrm{Y}$ eut-il d'autres raids de peu antérieurs à 252? Les Perses attaquèrent l'Empire romain dès 238/239 et s'emparèrent d'un certain nombre de villes, ce qui poussa Gordien III à conduire une campagne depuis Antioche pour reprendre les villes de Carrhes et de Nisibe. ${ }^{153}$ Zeugma fut-elle sur le chemin de cette reconquête? Les sources écrites ne le mentionnent pas. ${ }^{154}$ Les années 259/260 ont vu une armée perse se diriger vers Samosate et s'emparer sans doute encore une fois d'Antioche. ${ }^{155}$

Autrement dit, en théorie, les couches de destruction peuvent ne pas renvoyer forcément toutes au même épisode guerrier. Les "trésors" mis au jour dans les fouilles fournissent-ils des informations supplémentaires? L'équipe du PHI en a découvert 4(?), qui n'apportent guère d'élément pertinent. ${ }^{156}$ L'équipe franco-turque en a aussi mis au jour plusieurs dont l'interprétation reste délicate. Deux d'entre eux pourraient avoir été constitués avant 253. Les monnaies qui ont été trouvées dans l'US 12038 auraient pu être déposées entre 242 et $246 .{ }^{157}$ Ce trésor

M. H. Dodgeon et S. N. C. Lieu, The Roman eastern frontier and the Persian Wars, AD 226-363. A documentary history (London 1991) 50 et 361-62, n.10; Sartre (supra n.144) 968.

Scott dans PHI vol. 3, 190.

Abadie-Reynal (supra n.115) 524.

Zeugma IV, 223; Butcher dans PHI vol. 3, 90-91.

Zeugma IV, ibid.

Zeugma IV, 216-18, ce qui n'empêche pas les contremarques peut-être sous le règne de Trajan Dèce. Aylward dans PHI vol. 1, 30.

Dodgeon et Lieu (supra n.146) 38.

Sartre (supra n.144) 964-65.

Ibid. 975.

Butcher dans PHI vol. 3, 10-15. Les monnaies les plus récentes datent de Gordien III (trésors 1 et 2), de Trajan Dèce et de Trébonius Gallus (trésors 3 et 4). Cependant, l'interprétation des deux premiers trésors est complexe: le trésor 1, qui regroupe des monnaies n'ayant pas de valeur à Zeugma, pourrait avoir simplement été entreposé dans l'attente d'une occasion de réutiliser ces monnaies (Butcher dans PHI vol. 3, 10-14). Quant au trésor 2, il ne comporte que 8 monnaies de bronze (Butcher, ibid. 14) et à ce titre pourrait n'être que les restes d'une bourse; en plus, l'interprétation du contexte est douteuse (il peut s'agir de colluvion).

Zeugma IV, 197 (ensemble homogène de monnaies d'Edesse et de Carrhes constitué rapidement et de valeur relativement peu importante). 
provient de la couche de destruction du triclinium décoré par la mosaïque de Pasiphaé (maison de Poséidon), ${ }^{158}$ et peut-être de l'étage qui pouvait se trouver au-dessus de cette pièce. ${ }^{159} \mathrm{Il}$ faut noter qu'un trésor constitué de 2,500 tétradrachmes en argent a été mis au jour dans la partie domestique de la même maison. Les pièces les plus récentes datent de 253 apr. J.-C. ${ }^{160}$ Sur la même terrasse, dans la couche de démolition de la maison 4,161 on a trouvé (US 12534) un lot de 33 monnaies, qui se serait constitué progressivement et aurait été déposé pendant le règne de Philippe Ier, avec un terminus ante quem fixé à $249 .{ }^{162}$ En revanche, dans la même maison, un autre trésor (US 12543) comporte de nombreuses contremarques sur des monnaies de Zeugma frappées sous Philippe père et fils. ${ }^{163}$ Ce lot de monnaies aurait donc été constitué un peu plus tard, probablement autour des années 250/253.

Il n'est pas sûr que ces légers décalages chronologiques entre des "trésors" provenant d'une même maison aient un sens. En tout cas, ils permettent de vérifier que les trésors, peut-être plus anciens, ne correspondent pas à des destructions matérielles puisque dans ces maisons aucune trace significative de réparation n'a pu être observée. Ils ont simplement pu être entreposés et cachés ou oubliés quelques années avant l'attaque sassanide: leur propriétaire est-il parti en les oubliant, alors même que le trésor de l'US 12038 représente à peu près le montant mensuel de la solde d'un militaire? ${ }^{164}$ Est-il mort sans avoir pu transmettre cet argent? Ces "trésors" seraient donc plutôt l'expression de destins individuels soumis à des aléas que l'on peine à définir.

En revanche, les sommes plus importantes qui constituent les trésors les plus récents rendent compte de la brutalité et de la violence de l'épisode qui marque la fin de ces maisons et tendent à en souligner le caractère exceptionnel par rapport aux épisodes précédents. Il n'empêche que ces petits trésors, s'ils sont légèrement plus anciens, révèlent peut-être que les années qui précèdent le raid sassanide furent troublées. Si l'on exclut d'éventuelles attaques perses qui ne seraient pas évoquées par les textes et n'auraient pas provoqué de destructions matérielles perceptibles, on peut penser aux morts occasionnées par l'épidémie de "peste de Cyprien" 165 qui aurait pu décimer la population à partir de 251 et provoquer certaines de ces ruptures de mémoire dont témoigneraient les trésors. ${ }^{166}$ On peut aussi imaginer des troubles politiques liés aux différentes usurpations qui ont marqué le milieu du IIIème s.

Cette possible succession de menaces et d'événements ponctuels que les recherches sur le terrain peinent à reconstituer précisément auraient au moins le mérite de fournir peut-être un élément, parmi d'autres, expliquant l'apparente facilité avec laquelle la ville a été prise et pillée. La fin de Zeugma, au IIIème s., peut ne pas se résumer à un seul et unique épisode violent et doit être comprise peut-être comme la conclusion de difficultés répétées n'ayant guère laissé de traces archéologiques ni modifié le paysage urbain, même si on ne peut exclure que certains des remaniements immédiatement antérieurs à l'attaque sassanide, signalés par l'équipe du PHI (voir plus haut, p. 829), ne soient l'expression matérielle de cette période troublée, plutôt que de l'occupation des maisons par des militaires.

158 Abadie-Reynal dans Zeugma I, 205.

159 Zeugma V, 284-85.

160 Önal (supra n.131) VII. La valeur importante de ce trésor, ainsi qu'une datation un peu plus récente du moment où le trésor a été dissimulé, obligent à imaginer une histoire différente de celle du lot de monnaie provenant de l'US 12038.

161 Abadie-Reynal dans Zeugma I, 229 et 231.

162 Zeugma IV, 197-98.

163 Zeugma IV, 198.

164 Zeugma IV, 197.

165 G. Tate, Les campagnes de la Syrie du Nord du IIe au VIle siècle (BAH 133; Paris 1992) 301; E. Gozalbes Cravioto et I. García García, "Una aproximación a las pestes y epidemias en la Antigüedad," Historia Antigua 26 (2013) 76-77.

166 Sur les trésors que l'on doit retrouver en plus grand nombre en période d'épidémie, voir J. Durliat, "La peste du VIe siècle," dans C. Morrisson et J. Lefort (éds.), Hommes et richesses dans l'Empire byzantin, vol. 1: IVe-VIIe siècle (Paris 1989) 111. 


\section{L'économie}

\section{L'époque romaine}

Il est actuellement impossible de définir ce qu'a pu être la vie économique à Séleucie ou à Apamée à l'époque hellénistique: le matériel mis au jour pour cette période est trop rare ou n'a pu être suffisamment étudié. En revanche, pour l'époque romaine, le faciès économique de Zeugma peut se définir grâce à un matériel abondant. Cependant, l'accent est mis, en fonction des publications, sur des aspects différents de ces échanges. La publication du PHI souligne à plusieurs reprises que le site de Zeugma correspond, en particulier par le matériel céramique, à un profil plutôt mésopotamien, et que les courants d'échanges méditerranéens n'atteignent guère ce site. ${ }^{167}$ Cette hypothèse remet en question la conception de la ville traditionnellement développée, selon laquelle il s'agirait, avant tout, d'un important lieu de passage de l'Euphrate d'une route reliant la façade méditerranéenne à la Mésopotamie, voire au-delà. ${ }^{168}$

Il faut donc examiner précisément les différentes catégories de matériel sur lesquelles reposent les arguments. La thèse selon laquelle Zeugma s'insèrerait avant tout dans des réseaux régionaux propres à la vallée de l'Euphrate s'appuie, pour la fin de l'époque hellénistique et le Haut-Empire, sur la présence au Ier s. av. et apr. J.-C. de la Sigillée orientale A et de la céramique parthe glaçurée sur le site, ${ }^{169}$ ce qui permettrait de rapprocher le matériel de Zeugma de celui de sites comme Doura Europos ou Hama.

Cette affirmation se heurte à une évaluation précise de la céramique attestée sur le site, et en particulier de la céramique parthe. À Zeugma elle ne constitue qu'une infime partie du matériel: toujours moins de $1 \%$ du poids dans les différents groupes distingués par l'équipe du PHI. ${ }^{170}$ Contrairement à ce que l'on peut observer, par exemple, à Doura Europos ${ }^{171}$ ou même, plus près de Zeugma, dans les niveaux hellénistiques de Jebel Khalid, ${ }^{172}$ la céramique parthe glaçurée reste anecdotique. ${ }^{173}$ En revanche, la Sigillée orientale A est très fréquente à Zeugma, dans les groupes des Ier s. av. et apr. J.-C. Mais la domination de cette production est générale dans tout le Moyen-Orient de cette époque, que l'on se situe sur la côte, au Liban ou en Israël, ou à l'intérieur des terres, comme à Hama ${ }^{174}$ ou à Jebel Khalid. ${ }^{175}$ Autrement dit, la diffusion de la Sigillée orientale A est si générale que l'on ne peut rien en conclure sur les courants d'échanges; ${ }^{176}$ mais on peut déjà dire que, par la rareté de la céramique dite parthe, Zeugma offre un faciès différent de celui de Doura Europos et, peut-être dans une moindre mesure, de sites plus au nord dans la vallée de l'Euphrate, comme Jebel Khalid.

Aylward dans PHI vol. 1, 25, 27 et 29.

Kennedy (supra n.4) 11; Abadie-Reynal (supra n.11) 360-61.

Aylward dans PHI vol. 1, 29.

Kenrick dans PHI vol. 2, 8, 10 et 25.

N. Toll, The excavations at Dura Europos. Final reports, vol. IV, 1.1: green glazed ware (New Haven, CT 1943). Pour certaines réserves sur la quantité réelle de la céramique glaçurée à Doura-Europos voir H. Jackson, "The green-glazed wares from Jebel Khalid, 1986-2006," dans ead. et J. Tidmarsh, Jebel Khalid on the Euphrates, vol. 3. The pottery (Sydney 2011) 481 n.110.

Jackson ibid. 431: la céramique glaçurée parthe représenterait approximativement $1 \%$ de l'ensemble de la céramique mise au jour dans les niveaux antérieurs à 75 av. J.-C. de la seule insula à avoir été fouillée sur le site.

173 Dans l'ensemble des fouilles franco-turques, cette production représente moins de $0.4 \%$ de toute la céramique mise au jour, en nombre de fragments (données inédites).

4 J. Lund, "A fresh look at the Roman and Late Roman fine wares from the Danish excavations at Hama, Syria," dans H. Meyza et J. Młynarczyk (éds.), Hellenistic and Roman pottery in the eastern Mediterranean. Advances in scientific studies (Varsovie 1995) 136-37; D. Malfitana, "Eastern terra sigillata wares in the eastern Mediterranean. Notes on an initial quantitative analysis," dans F. Blondé, P. Ballet et J.-F. Salles (éds.), Céramiques hellénistiques et romaines. Productions et diffusion en Méditerranée orientale (TMO 35; Lyon 2002) 146-51.

J. Tidmarsh, "The imported fine wares at Jebel Khalid," dans Jackson et id. (supra n.171) 326-28.

Pourtant, Kenrick (PHI vol. 2, 75) en tire argument pour mentionner les contacts de Zeugma avec la région côtière d'Antioche, contredisant ainsi Aylward, PHI vol. 1, 29. 
Un autre argument est avancé pour confirmer cet isolement supposé durable de Zeugma par rapport à la façade méditerranéenne au cours de la première moitié du IIIème s. La publication du PHI souligne le nombre limité d'importations de céramique fine et l'importance des productions régionales à cette époque. ${ }^{177}$ Cet argument, une fois de plus, doit être mis en perspective à la lumière de la situation d'autres sites. Cette période, de façon générale, ne voit que peu d'importations de céramiques fines au Moyen-Orient, quels que soient les sites considérés, qu'ils soient côtiers ou de l'intérieur. ${ }^{178}$ Cependant, au milieu du IIIème s. on assiste au début des importations, en nombre significatif, des sigillées claires africaines, en particulier de la formes Hayes $50 .{ }^{179}$ Ce phénomène est perceptible à Zeugma comme ailleurs. ${ }^{180}$ Le site ne se différencie donc guère, pour les importations de céramiques fines, des autres sites de la Syrie romaine.

Les travaux sur les amphores, de leur côté, permettent de relier Zeugma aux échanges méditerranéens. Certes, beaucoup de jarres sont de fabrication locale ou régionale. Pourtant, si pendant le Haut-Empire on ne trouve qu'un nombre restreint d'amphores méditerranéennes à Zeugma (mais les groupes de céramique de cette période sont trop limités pour être significatifs), à partir du IIIème s. on voit se développer les importations lointaines d'amphores qui permettent de mettre Zeugma en relation avec les courants d'échanges méditerranéens. On a pu mettre au jour sur le site des amphores campaniennes de type coen, ${ }^{181}$ mais aussi des amphores de Bétique (principalement Dressel 20) apportant l'huile de la vallée du Guadalquivir. ${ }^{182}$ À côté de ces importations occidentales, on trouve surtout des produits égéens en nombre tout à fait significatif, en particulier des amphores de Cos, des amphores Kapitän II et des variantes anciennes des amphores LRA3, monoansées. ${ }^{183}$ Ce faciès de matériel montre un schéma d'approvisionnement différent de ce que l'on trouve sur la côte libanaise. Il est dommage de ne pas mieux connaître le matériel mis au jour à Antioche, mais on peut supposer que c'est surtout par ce port que sont passées ces importations lointaines, qui sont aussi attestées sur (ou près de) la côte méditerranéenne, à Chypre et à Tarse, par exemple. ${ }^{184}$ Ces rapports sans doute étroits avec Antioche sont aussi attestés par l'étude des monnaies de Zeugma: la publication des fouilles du PHI montre que les monnaies d'Antioche sont surtout présentes aux Ier et IIème s. puisqu'elles sont supplantées, ensuite, par les émissions mésopotamiennes d'Edesse, en particulier à partir du règne de Gordien III,' 185 quand l'atelier produisant les grands bronzes de

Aylward ibid. 27.

Pour l'absence généralisée de céramiques fines importées au Moyen-Orient, voir, par exemple, pour Hama, Lund (supra n.174) 145; pour Anemurium, ibid. 161 et pl. 11.1-4; pour Apamée ou Jérusalem, id., "Centuries of darkness? A ceramic sidelight on Cyprus and the eastern Mediterranean between about A.D. 200 and A.D. 350," dans P. Åström (éd.), Acta Cypria (Jonsered 1992) vol. 2, 193-213; pour la région de Palaipaphos, id., "Pottery of the Classical, Hellenistic and Roman periods," dans L. W. Sørensen et D. Rupp (éds.), The land of the Paphian Aphrodite, The Canadian Palaipaphos Survey Project, vol. 2 (SIMA 104:2; Göteborg 1993) 138-39; Malfitana (supra n.174) 155.

M. Bonifay, "Observations sur la diffusion des céramiques africaines en Méditerranée orientale durant l'Antiquité tardive," dans Baratte et al. (supra n.115) 566; P. Reynolds, "Transport amphorae of the first to seventh centuries: early Roman to Byzantine periods," dans PHI vol. 2, 99 (pour Beyrouth).

Abadie-Reynal (supra n.115) 524-25; M. Gschwind, "Roman pottery from Zeugma: continuity, change, and the Central Euphrates perspective," dans Ergeç (supra n.39) 59.

C. Abadie-Reynal, "Les amphores méditerranéennes d'importation trouvées à Zeugma: présentation préliminaire," dans J. Eiring et J. Lund (éds.), Transport amphorae and trade in the eastern Mediterranean (Monog. Danish Inst. Athens 5, 2004) 20; Reynolds dans PHI vol. 2, 98.

Reynolds dans PHI vol. 2, 98.

Abadie-Reynal (supra n.181) 16-17; Aylward dans PHI vol. 1, 25; Reynolds dans PHI vol. 2, 99.

J. W. Hayes, Paphos III: the Hellenistic and Roman pottery (Nicosie 1991) 92-95, 154, 207 et 209 ; F. F. Jones, "The pottery," dans H. Goldman (éd.), Excavations at Gözlü Kule, Tarsus vol. I (Princeton, NJ 1950) $274, n^{\circ} 797,278, n^{\circ} 834$, par exemple.

Kenrick dans $P H I$ vol. 2, 18-19. 
type grec d'Antioche ferme. ${ }^{186}$ Les fouilles franco-turques permettent de compléter ce tableau: les monnaies d'Antioche y sont aussi attestées dans les niveaux du IIIème s., et pendant le règne de Sévère Alexandre elles dominent encore le matériel numismatique, ${ }^{187}$ avec les monnaies d'Edesse.

Le matériel mis au jour et daté du Haut-Empire reste trop limité quantitativement pour permettre une interprétation de type économique des différentes tendances, d'autant plus que la place à attribuer à la présence de la légion à Zeugma reste difficile à apprécier. Les liens de Zeugma avec la façade méditerranéenne apparaissent, en revanche, bien réels quand le matériel devient plus abondant pendant la première moitié du IIIème s. Les produits méditerranéens ne dominent pas le marché local, mais ils sont régulièrement présents et attestent sans doute de relations avec la région d'Antioche. On ne peut donc pas parler d'isolement économique de Zeugma. ${ }^{188}$ D'ailleurs, il faut mentionner la trouvaille exceptionnelle de milliers d'empreintes de sceaux, faite par l'équipe turque ${ }^{189}$ lors de la fouille d'un bâtiment d'archives, sans parler des exemplaires trouvés en contextes d'habitat. ${ }^{190}$ Ce matériel considérable, qui est daté entre le Ier s. av. J.-C. et le début du IIIème s. apr. J.-C., ${ }^{191}$ atteste du dynamisme des transactions qui se déroulent à Zeugma, et peut-être, entre autres, des exemptions de taxes. ${ }^{192}$ Cette découverte reste difficile à interpréter, mais elle montre bien que la ville est durablement impliquée dans des opérations d'envergure et ne vit pas repliée sur elle-même.

Il est d'ailleurs dommage qu'une analyse globale et détaillée de la vie économique à Zeugma, conduite par la publication du PHI, ne pousse guère au-delà du milieu du IIIème s. ${ }^{193}$ que pour quelques catégories particulières d'objets. En effet, certaines tendances qui commencent à s'affirmer dans la première moitié du IIIème s. sont peut-être les prémices de changements économiques importants qui parcourent le bassin méditerranéen pendant cette période et dont le site de Zeugma se fait l'écho: l'étude du matériel tardif de Zeugma renforce l'idée d'un site alimenté en partie par des réseaux d'échanges méditerranéens (voir plus bas, p. 841-43).

D'autres types d'échanges sont attestés à Zeugma et soulignés par la publication du PHI: ils se feraient le long de la vallée de l'Euphrate, du nord jusqu'au sud, à Doura Europos, en passant par d'autres vallées intérieures de même orientation. ${ }^{194}$ Pour examiner cette question, il faut distinguer les échanges régionaux qui se font souvent le long du fleuve (mais cela concerne avant tout des productions de céramique commune, originaires, au plus de quelques dizaines de kilomètres), des échanges à plus longue distance nord-sud, qui sont attestés de façon générale dans les publications des deux équipes mais avec des nuances importantes dans leur matérialisation et leur interprétation. L'équipe du PHI s'appuie avant tout sur la céramique pour définir ces échanges, en mettant l'accent sur une communauté de matériel à notre avis partiellement fausse et surinterprétée (voir plus haut, p. 838) entre les sites de Zeugma, de Hama et de Doura Europos, dont l'unité serait basée sur la présence de la Sigillée orientale A et de la céramique parthe. Ces échanges pourraient aussi venir de Beyrouth, ${ }^{195}$ alors même que la différence de faciès entre la céramique du IIIème s. de Beyrouth et de Zeugma est

Zeugma IV, 207.

Zeugma IV, 206; voir aussi à ce propos Önal (supra n.131) p. VI-VII, même si ce trésor ne se prête pas à une interprétation d'ordre économique.

Contra Aylward dans PHI vol. 1, 25.

Suivant les publications, les nombres varient mais restent de toute façon impressionnants: 65,000 empreintes dans M. Önal. Clay impressions of Zeugma (Gaziantep 2007) 8 pour le bâtiment des archives seul, 100,000 pour Aylward PHI vol. 1, 25, plus de 140,000 pour S. Herbert, "Bullae," dans PHI vol. 2, 211.

Herbert dans PHI vol. 2, 212.

Önal (supra n.131) 14.

Voir à ce sujet Herbert dans PHI vol 2, 211.

Aylward dans PHI vol. 1, 24-25.

Ibid. 25 et 41, n.276 pour l'époque sévérienne.

Ibid. 25. 
patente. ${ }^{196}$ L'existence donc d'échanges commerciaux, au-delà de l'échelle régionale, le long de la vallée de l'Euphrate ne paraît guère confirmée. L'équipe franco-turque est plutôt sensible à l'existence de relations avec la mer Noire, qui se matérialisent par la présence de monnaies du Pont en nombre significatif ${ }^{197}$ au cours du IIIème s., aussi attestées dans les fouilles du PHI. ${ }^{198}$ Cependant il semble peu probable que l'arrivée de ces monnaies soit le résultat de transactions commerciales. L'époque troublée dans laquelle semble s'inscrire ce numéraire (Septime Sévère) permet de l'associer avec plus de vraisemblance aux mouvements de troupes rapatriées depuis le nord, vers la frontière orientale de l'Empire. Finalement, pendant la première moitié du IIIème s. la plus grande partie de la vie économique se déroulerait certes, dans le cadre restreint de la région (ce qui caractérise l'ensemble du Moyen-Orient de cette époque). ${ }^{199}$ Cependant, cela ne doit pas faire oublier que Zeugma se trouve sur une grande route ouest-est et que, à ce titre, les grandes tendances du commerce méditerranéen se retrouvent dans cette ville, de façon amortie, mais néanmoins tout à fait perceptible.

\section{L'époque romaine tardive et protobyzantine}

Quand on aborde la vie économique de Zeugma dans l'Antiquité tardive, la conception d'une ville n'ayant que peu de contacts au-delà de sa région apparaît encore moins justifiée. Certes, la quantité de matériel céramique tardif publiée par le PHI reste relativement limitée. Seuls les groupes $\mathrm{E}$ et $\mathrm{F}$, datés du début du VIème et du début du VIIème s., apportent une quantité significative, mais restreinte, de matériel, ce qui ne permet pas de travailler sur le IVème et sur une grande partie du Vème s. Il est donc difficile, à partir de cette publication et de la sélection qui y a été opérée par suite de contraintes diverses, de définir le fonctionnement de la vie économique de Zeugma pendant toute cette période. Pourtant, il apparaît, au VIème comme au début du VIIème s., que la céramique fine, de façon générale, est plus abondante qu'au IIIème s., et surtout qu'elle est presque totalement importée du domaine méditerranéen, que ce soit de la région de Phocée, de Chypre ou d'Afrique du Nord. ${ }^{200}$ Les groupes publiés par le PHI, bien que de dimensions différentes, laissent tous deux deviner une présence significative des céramiques fines méditerranéennes: dans le groupe $\mathrm{F}$ (début du VIIème s.) la sigillée phocéenne représente env. $13 \%$ du nombre total de fragments, toutes céramiques confondues. ${ }^{201}$ Les mêmes constatations peuvent être faites à partir d'autres fouilles. ${ }^{202}$ On peut ajouter qu'à Zeugma la sigillée phocéenne représente un peu moins de $70 \%$ de l'ensemble de la céramique fine tardive 203 mise au jour dans les fouilles franco-turques, ce qui confirme, globalement, l'importance de cette production dans la Syrie intérieure tardive. Faute de publication exhaustive et précisément quantifiée de ce matériel, on ne peut guère distinguer le faciès du matériel de Zeugma de celui d'autres sites intérieurs de la Syrie comme Resafa ou Hama. À vrai dire, la distribution des vases en sigillée phocéenne paraît être assez homogène entre sites côtiers et sites de l'intérieur. ${ }^{204}$ La présence de sigillées claires africaines est aussi relativement bien attestée à Zeugma pendant toute la période où ces productions ont été exportées en Méditerranée; sur ce

196 Reynolds dans PHI vol. 2, 98-100.

197 Frascone dans Zeugma IV, 205-7 et 225-28.

198 Kenrick dans PHI vol. 2, 7.

199 Gschwind (supra n.80) 59.

200 Kenrick dans PHI vol. 2, 52 (groupe E).

201 Ibid. 57 (groupe F).

202 Gschwind (supra n.80) 62-63.

203 Abadie-Reynal (supra n.115) 523; contra, Reynolds dans PHI vol. 2, 105. Huit exemplaires de sigillée phocéenne sont publiés dans les fouilles du PHI, contre presque $600 \mathrm{mis}$ au jour dans les fouilles franco-turques. Cette différence dans la quantité de matériel considéré explique les appréciations contradictoires portées sur l'importance de cette production.

204 J. W. Hayes, "Late Roman fine wares and their successors: a Mediterranean Byzantine perspective (with reference to the Syro-Jordanian situation)," dans E. Villeneuve et P. Watson (éds.), La céramique byzantine et proto-islamique en Syrie-Jordanie (IV s.-VIII s. apr. J.-C.) (BAH 159; Beyrouth 2001) 279; Bonifay (supra n.179) 566-72 et, avec des conclusions proches obtenues à partir du matériel africain de Zeugma, Abadie-Reynal (supra n.115) 534-36. 
site elles semblent faire écho aux fluctuations perçues dans l'ensemble de la région. ${ }^{205} \mathrm{Il}$ serait intéressant, de ce point de vue, de situer Zeugma parmi les autres sites intérieurs de la Syrie, en comparant les quantités de sigillées claires africaines attestées; leur présence plus importante pourrait signaler l'existence de liens particulièrement étroits avec la façade méditerranéenne, mais à l'heure actuelle peu de données sont exploitables. À Zeugma, dans les fouilles francoturques, ces productions représentent globalement un peu moins de 1/5ème des céramiques fines de table pendant la période qui va du milieu du IIIème au VIIème $s .{ }^{206}$ Ces chiffres paraissent effectivement légèrement supérieurs à ceux que l'on peut observer sur d'autres sites: env. $11 \%$ de l'ensemble des céramiques fines importées à Resafa, ${ }^{207}$ où la sigillée africaine est considérée comme relativement rare. ${ }^{208}$

Cela dit, il faudrait nuancer ces comparaisons et tenir compte des fluctuations observées dans les importations en fonction des périodes d'occupation des sites. ${ }^{209}$ De façon générale, sur des sites plus proches de la Méditerranée les proportions semblent changer: à Hama, les sigillées africaines paraissent dominer, ${ }^{210}$ tandis qu'à Antioche la publication ancienne ne permet guère que des considérations générales où, pourtant, les productions africaines tiennent une part importante qui pourrait approcher des $40 \%$ de l'ensemble de la céramique fine importée. Malgré des estimations le plus souvent approximatives, il semble que la présence des sigillées claires africaines est d'autant plus forte que l'on se trouve près des côtes, ce qui est logique. Cela dit, Zeugma semble compter une présence relativement plus importante de sigillées africaines que Resafa, dont la situation en bordure de l'Euphrate est à peu près similaire. La route est-ouest qui traverse l'Euphrate participe sans doute, dans une certaine mesure, au développement des échanges avec la zone méditerranéenne.

La présence de la sigillée chypriote tardive peut permettre d'avancer dans la réflexion. D'après la publication du PHI, cette production semble assez peu présente dans les groupes du début du VIème et du début du VIIème $s .{ }^{211}$ En revanche, si l'on considère l'ensemble du matériel tardif des fouilles franco-turques, ces vases représentent environ la même quantité de fragments que la production africaine. Cette différence d'appréciation résulte clairement de la prise en compte dans les fouilles franco-turques de groupes du Ve s.: c'est avant tout à cette période qu'appartiennent les formes représentées à Zeugma. Ces caractéristiques de l'approvisionnement du site, d'autre part, permettent de revoir l'hypothèse selon laquelle les importations méditerranéennes parviendraient à Zeugma par la voie fluviale méridionale depuis Beyrouth. ${ }^{212}$ En effet, sur ce site, la sigillée chypriote tardive reste importante jusqu'au VIIème s. ${ }^{213}$ alors qu'à Antioche (pour le peu que l'on en sache) les formes tardives semblent absentes. ${ }^{214}$ Cette différence dans l'approvisionnement d'Antioche et de Beyrouth permettrait de rapprocher le premier site de ce que l'on peut observer à Zeugma, et donc de confirmer l'importation de la sigillée chypriote tardive par Séleucie de Piérie.

Les amphores tardives apportent des informations complémentaires sur la place qu'occupe le commerce à longue distance à Zeugma. Evidemment, les amphores peintes tardives, dont $\mathrm{P}$. Reynolds présente la première typologie précise ${ }^{215}$ dominent le marché local; ces amphores

Bonifay ibid. 566-72.

Abadie-Reynal (supra n.115) 523.

Hayes (supra n.204) 281.

M. Mackensen, Resafa, vol I. Eine befestigte spätantike Anlage vor den Stadtmauern von Resafa (Mayence 1984) 41.

Bonifay (supra n.179) 566-70.

Lund (supra n.174) 139-43; repris par Hayes (supra n.204) 279.

Kenrick dans PHI vol. 2, 52-53 et 56-57.

Aylward dans PHI vol. 1, 25.

Reynolds dans PHI vol 2, 105; voir aussi id., "Fine wares from Beirut contexts," dans M. Ángel Cau, P. Reynolds et M. Bonifay (éds.), LRFW1. Late Roman Fine Wares. Solving problems of typology and chronology (Oxford 2011) 224-30.

J. W. Hayes, Late Roman pottery (Londres 1972) 382 (à propos de la forme 9).

Reynolds dans PHI vol. 2, 112-17. 
ont donné lieu à des analyses qui montrent qu'elles ne sont sans doute pas originaires des alentours de Zeugma, mais de la moyenne vallée de l'Euphrate. ${ }^{216}$ Sans doute est-ce en partie sur ce matériel que s'appuie $W$. Aylward pour mettre en avant le réseau économique restreint de Zeugma.

Pourtant, cette appréciation appelle quelques remarques. La situation à Zeugma n'est guère différente de celle que l'on peut observer ailleurs à la même époque, y compris le long des côtes où les productions locales ou régionales d'amphores peuvent être importantes. ${ }^{217}$ Outre la présence de céramiques fines importées, il existe aussi à Zeugma des importations relativement lointaines de produits alimentaires. Les amphores africaines sont rares, ce qui ne doit pas surprendre puisqu'il s'agit là d'un trait commun à l'ensemble du bassin oriental de la Méditerranée, ${ }^{218}$ même si quelques exemples ont été repérés dans les fouilles franco-turques. ${ }^{219}$

En revanche, la présence des grands types d'amphores orientales tardives mérite sans aucun doute une évaluation plus précise que ce que nous apporte la publication du PHI, qui d'ailleurs donne des appréciations un peu différentes selon les chapitres. ${ }^{220}$ Dans les fouilles franco-turques, les amphores peintes régionales représentent, en nombre de fragments, un peu moins de $80 \%$ du total du matériel amphorique tardif (pas encore publié). La majeure partie des autres amphores (env. 22\%) sont d'origine orientale. Les plus fréquentes sont les LRA1 (Chypre, Cilicie) dont nous avons fourni une première évaluation de l'importance dans les fouilles franco-turques: ${ }^{221}$ elles représentent à elles seules env. 9\% de tout le matériel amphorique tardif. Elles sont suivies de près par les amphores LRA3 (région d'Éphèse), tandis que les amphores LRA4 (Gaza) forment un peu moins de 5\% des lots d'amphores. Le restant est constitué par quelques amphores LRA5, quelques amphores égéennes et africaines ou occidentales, et, enfin, par des amphores de Sinope (voir plus bas, p. 844-45). D'autre part, la provenance de ces amphores, et en particulier de celles qui sont les mieux représentées (LRA1 et LRA3), confirme ce que l'importance des sigillées phocéennes et chypriotes soulignait déjà: ces échanges se font avant tout selon une route ouest-est, en provenance de Séleucie de Piérie et Antioche, et non pas, comme il est suggéré par la publication du PHI, sur un axe nord-sud, le long des vallées du Habur, du Balih et du Quwaiq. ${ }^{222}$ Les échanges avec la façade méditerranéenne et le bassin égéen sont réguliers et représentent une quantité significative de matériel, sans être prédominants.

Ces mouvements ouest-est peuvent aussi être perçus grâce aux monnaies. L'équipe du PHI n'a trouvé qu'un nombre limité de monnaies tardives; ${ }^{223}$ les fouilles franco-turques permettent,

216 Ibid. 113-14 et C. Doherty, "Petrographic analysis of transport amphorae," dans PHI vol. 2, 165-67. Ces deux contributions sur le sujet ne s'accordent d'ailleurs pas vraiment: alors que les analyses de pâte montrent une grande homogénéité dans leur composition, P. Reynolds distingue, à partir de l'aspect de la pâte, mais aussi de particularités dans les formes et la décoration, deux groupes qui pourraient correspondre à deux régions de production différentes. L'une de ces productions (forme 14) serait courante à Zeugma, mais rare à Resafa.

217 Pour Beyrouth où la production d'amphores locales est importante, voir P. Reynolds, "Beirut in the Byzantine period: 4th-7th centuries," dans Ch. Bakirtzis, Actes du VIIe Congrès int. sur la Céramique Médiévale en Méditerranée, 1999 (Athènes 2003) 538-39.

218 Voir à ce sujet Bonifay (supra n.179) 572-77.

219 Abadie-Reynal (supra n.11) 20, fig. 9. Voir aussi, par exemple, ZAP 14142.34 ou une dizaine d'autres fragments non publiés.

220 Aylward dans PHI vol. 1, 25 (repliement économique); Reynolds dans PHI vol. 2, 105: "Zeugma in the seventh century also benefited from a regular and somewhat plentiful supply of long-distance imports from the Black Sea (Sinope fish sauce amphorae) and LRA1 amphorae from Cilicia and possibly Cyprus, with rarer Palestinian imports".

221 Abadie-Reynal (supra n.11) 17-19.

222 Aylward dans PHI vol. 1, 25; contra Reynolds, ibid. vol. 2, 105, où toute l'importance d'Antioche et de son port est soulignée. 
par la quantité mise au jour, de compléter les données. ${ }^{224}$ Elles montrent, d'une part, que les monnaies romaines tardives "constituent une part importante du numéraire", 225 et que, malgré la présence d'ateliers variés, les frappes d'Antioche dominent et deviennent majoritaires à partir du règne de Constantin Ier, pour le rester à l'époque byzantine. ${ }^{226}$ Antioche et son port Séleucie de Piérie semblent donc bien être des centres de redistribution, dont l'extension vers l'est est actuellement difficile à préciser. ${ }^{227}$

Pourtant, l'importance relative à Zeugma des sigillées claires africaines et des importations venant du bassin égéen et de Chypre (ou de la côte cilicienne) laisse à penser que, au moins le long de la route qui franchit l'Euphrate à Zeugma, ce centre économique joue un certain rôle, même si (à part Zeugma) l'absence de site fouillé sur cette route empêche d'approfondir la question. Cela dit, les différences sensibles entre le matériel de Zeugma et celui de Resafa ou d'autres sites continentaux, comme Qusair as-Saila, permettent de compléter le dossier. La sigillée phocéenne, partout très abondante au Moyen-Orient, ne peut servir d'élément discriminant. En revanche, la présence des sigillées claires africaines, de la sigillée chypriote tardive, ainsi que de certaines catégories d'amphores comme les LRA1 et LRA3, absentes de Resafa, ${ }^{228}$ constituent des éléments plus significatifs et permettent de rétablir Antioche dans son rôle de centre de redistribution. ${ }^{229}$

Outre ces relations est-ouest avec la façade méditerranéenne, dont il faudrait préciser les modalités exactes (commerce? rôle des armées? de l'annone?), existe-t-il aussi des traces de circulation de marchandises sur la longue distance, suivant un axe fluvial sud-nord, ${ }^{230}$ qui expliquerait le nombre relativement important des amphores de Sinope au VIIème s.? ${ }^{231}$ Cette hypothèse, peu vraisemblable, impliquerait que ces importations arrivaient par la côte à Beyrouth, puis, de là, empruntaient les vallées de l'intérieur pour parvenir à Zeugma. Cependant, le matériel de Beyrouth diffère de celui de Zeugma, pour les céramiques fines (voir plus haut, p. 842) mais aussi pour les amphores de Sinope, ${ }^{232}$ qui semblent bien mieux représentées à Zeugma qu'à Beyrouth. Pour être plus précis, la publication du PHI montre qu'elles sont présentes dans tous les contextes du VIIème s. pris en compte, ${ }^{233}$ à peu près à égalité avec les amphores LRA1. Dans les fouilles franco-turques, elles apparaissent aussi, ${ }^{234}$ mais en quantité moins importante que les amphores LRA1, LRA3 et LRA4 (publication à paraître). Elles sont absentes de Resafa et des sites situés à l'est d'Alep. ${ }^{235}$ Seuls quatre exemplaires proviendraient de Dibsi Faraj. En revanche, elles apparaissent en nombre à Séleucie de Piérie et Ibn Hani. ${ }^{236}$ Ces amphores à pâte claire de Sinope suivent donc sans doute un autre cheminement que celui qui est proposé dans la publication du PHI; la plupart des sites intérieurs ne reçoivent guère ces importations, qui ont avant tout une distribution côtière, comme les amphores

\section{Zeugma IV, 207-11. \\ Zeugma IV, 208. \\ Zeugma IV, 211.}

N. Haidar Vela et D. Piéri, "Faciès céramique de la Syrie du Nord protobyzantine," dans G. Charpentier et V. Puech (éds.), Villes et campagnes aux rives de la Méditerranée ancienne. Hommages à Georges Tate (Topoi, Suppl. 12, 2013) 126.

Reynolds dans PHI vol. 2, 105.

Un rôle que Reynolds lui reconnait pleinement dans $P H I$ vol. 2, 105, contrairement à ce que l'on peut lire dans Aylward ibid. 25.

Aylward ibid.

Ibid.

Reynolds dans PHI vol. 2, 105.

Ibid. 100-2.

Abadie-Reynal (supra n.181) 20.

Reynolds dans PHI vol. 2, 105.

D. Kassab Tezgör, S. Lemaître et D. Piéri, "La collection d'amphores d'Ismail Karakan à Sinop," AnatAnt 11 (2003) 178; D. Kassab Tezgör, "Le réseau commercial des amphores sinopéennes," dans id. et N. Inaishvili (éds.), PATABS. Production and trade of amphorae in the Black Sea (Varia Anatolica 21; Istanbul 2010) 171-72. 
égéennes. Leur présence à Zeugma peut peut-être être associée aussi au flux monétaire dominant sur le site au VIème et au début du VIIème s.: il est composé de monnaies d'Antioche et de Constantinople; peu perceptible dans les fouilles du PHI, ${ }^{237}$ il apparaît clairement à partir du matériel des fouilles franco-turques. ${ }^{238}$ Les importations de garum venant de la Mer noire et transitant par Constantinople pourraient rejoindre le long des côtes de l'Anatolie les transports de sigillées phocéennes et d'amphores LRA3 pour gagner le sud du bassin égéen, puis la Cilicie ou Chypre, avant d'atteindre Séleucie et la région d'Antioche.

Cette grande voie maritime a depuis longtemps été repérée, ${ }^{239}$ quelles que soient les modalités qui président à ces échanges. Faut-il associer ces importations assez spécifiques à la présence militaire sur le limes oriental? On connaît le poids des armées aux VIème-VIIème s., qui est très souvent évoqué. ${ }^{240}$ Pourtant, comme le montre en Syrie du nord un site comme Resafa (voir p.. 842, 844), ces échanges lointains ne sont perçus que de façon très atténuée à l'intérieur des terres, y compris sur les sites à vocation militaire. De ce point de vue, Zeugma se distingue des autres sites de la vallée de l'Euphrate: les importations y sont plus présentes et suivent en général les fluctuations sensibles sur les sites côtiers et en particulier à Antioche/Séleucie de Piérie. Sans doute, cette sensibilité aux grands échanges maritimes, particulièrement perceptible à l'époque romaine tardive et à l'époque protobyzantine, peut-elle être interprétée comme une conséquence de la circulation le long de la route qui relie la façade méditerranéenne à la Mésopotamie et qui traverse l'Euphrate à Zeugma. Si l'on ignore quelles importations orientales servaient de contrepartie à ces flux méditerranéens, cela ne signifie pas qu'elles n'existaient pas; de même, faute de sites explorés à l'est de Zeugma, on ne sait pas quelle part de ces produits méditerranéens était avant tout destinée à la consommation des habitants de Zeugma et jusqu'où ces importations étaient acheminées à l'est de l'Euphrate.

Beaucoup de questions subsistent. Il serait important de reprendre, sans a priori, l'ensemble des questions évoquées ici en s'appuyant sur du matériel archéologique suffisamment abondant pour avoir du sens, et surtout sur des groupes ou des contextes représentant toutes les périodes couvertes par l'histoire de Zeugma. Ce n'est qu'à ce prix que les transitions, les évolutions, les continuités et les ruptures dans les approvisionnements, aussi bien que dans l'histoire du tissu urbain ou la présence de l'armée, pourront être saisies. Des pistes d'explication surgiront de ces données, mais pour comprendre véritablement les phénomènes décrits il faudrait aussi arriver à rattacher Zeugma à un réseau de sites fouillés et publiés dans la région - autant dire que beaucoup de temps sera encore nécessaire avant de pouvoir replacer Zeugma dans un schéma historique et explicatif satisfaisant.

abadiereynal@wanadoo.fr

CNRS-UMR 5189-Hisoma, Université Lumière-Lyon 2

\footnotetext{
237 Butcher dans PHI vol. 3, 42-43.

238 Zeugma IV, 211.

239 C. Abadie-Reynal, "Céramique et commerce dans le bassin égéen du IV e au VII ${ }^{\mathrm{e}}$ siècle," dans Morrisson et Lefort (supra n.166) 158-59, fig. 17.

240 Haidar Vela et Piéri (supra n.227) 126-27.
} 